MATHEMATICS OF COMPUTATION

Volume 81, Number 278, April 2012, Pages 661-685

S $0025-5718(2011) 02526-3$

Article electronically published on July 20, 2011

\title{
GALERKIN AND STREAMLINE DIFFUSION FINITE ELEMENT METHODS ON A SHISHKIN MESH FOR A CONVECTION-DIFFUSION PROBLEM WITH CORNER SINGULARITIES
}

\author{
SEBASTIAN FRANZ, R. BRUCE KELLOGG, AND MARTIN STYNES
}

\begin{abstract}
An error analysis of Galerkin and streamline diffusion finite element methods for the numerical solution of a singularly perturbed convectiondiffusion problem is given. The problem domain is the unit square. The solution contains boundary layers and corner singularities. A tensor product Shishkin mesh is used, with piecewise bilinear trial functions. The error bounds are uniform in the singular perturbation parameter. Numerical results supporting the theory are given.
\end{abstract}

\section{INTRODUCTION}

Consider the singularly perturbed boundary value problem

$$
\begin{aligned}
-\varepsilon \Delta u-p u_{x}+q u & =f & & \text { on } \Omega=(0,1)^{2}, \\
u & =0 & & \text { on the boundary } \partial \Omega,
\end{aligned}
$$

where the functions $p, q$ and $f$ are arbitrarily smooth and satisfy $p \geq \beta>0$ for some constant $\beta$, and $q>0$. The parameter $\varepsilon$ lies in the interval $(0,1]$. Since $q>0$ and the boundary data is continuous on $\partial \Omega$, the problem has a unique classical solution $u$ that lies in $C^{2, \alpha}(\Omega) \cap C(\bar{\Omega})$, where $C^{2, \alpha}(\Omega)$ is the standard Hölder space.

The solution $u$ of (1.1) usually has boundary layers on three sides of the square $\bar{\Omega}$ : an exponential outflow layer along $x=0$ and parabolic boundary layers along the tangential flow boundaries $y=0$ and $y=1$. In addition, if the right-hand side and the zero boundary data are not compatible with the differential equation at a corner, the solution has a singularity at the corner. These solution phenomena have been widely studied in the literature; see for example [10] for the boundary layers and [5, 6] for the corner singularities.

Analyses of Galerkin and streamline diffusion finite element methods for (1.1) that used bilinears on a tensor-product Shishkin mesh were given in [3, 4; see also [8, 12, 13, and their references, where analogous methods for a related problem that

Received by the editor September 3, 2009 and, in revised form, November 2, 2010 and January $25,2011$.

2010 Mathematics Subject Classification. Primary 65N30.

The research of the first author was supported by Science Foundation Ireland under the Research Frontiers Programme 2008; Grant 08/RFP/MTH1536.

The research of the second author was supported by the Boole Centre for Research in Informatics at National University of Ireland, Cork.

(C)2011 American Mathematical Society Reverts to public domain 28 years from publication 
has exponential layers but no parabolic layers were investigated. The hypotheses in all of these papers exclude corner singularities.

In the present paper we examine similar numerical methods, but minimal corner compatibility assumptions are made on the data so that at each corner one can have the classical $r^{2} \ln r$ singularity. The complicated interaction of these corner singularities with the boundary layers was described in [6] for constant $p$ and $q$. The corner singularities introduce fresh difficulties that did not arise in [3, 4, 8, 12, 13. and affect the choice of the streamline diffusion parameter.

Our mesh is the same as that of $[3,4$. Related meshes appeared in $8,12,13$. Thus the mesh is designed to handle exponential and parabolic boundary layers, but is not modified to fit the corner singularities.

Our aim is to devise a numerical method whose accuracy is guaranteed independently of the value of the singular perturbation parameter $\varepsilon$; thus, in our analysis we state explicitly all dependence on $\varepsilon$.

The paper is structured as follows. In Section 2 we give a decomposition of the solution $u$ that reveals the precise nature of the layers and singularities that it contains. Section 3 constructs a tensor-product Shishkin mesh suited to (1.1). The accuracy of piecewise bilinear interpolation on this mesh is analysed in Section 4 Section 5 analyses a Galerkin finite element method that uses bilinears on the same mesh; this section is subdivided by treating separately the diffusion and convection terms that appear in the associated bilinear form. It is shown that, measured in an $\varepsilon$-weighted energy norm, the Galerkin finite element solution attains the same order of accuracy as the bilinear nodal interpolant. Section 6 extends this analysis to the streamline diffusion finite element method and investigates how the presence of the corner singularities influences the choice of the stabilising parameters in this method. Finally, Section 7 describes some numerical experiments that verify the accuracy of our two finite element methods.

Notation. Throughout the paper $C$ denotes a generic constant that is independent of $\varepsilon$ and any mesh, and can take on different values at different points in the argument. For all measurable sets $\omega \subset \Omega$, let $(\cdot, \cdot)_{\omega}$ denote the inner product in $L_{2}(\omega)$ and let $\|\cdot\|_{0, \omega}$ denote the norm in $L_{2}(\omega)$. When $\omega=\Omega$ we simply write $(\cdot, \cdot)$ and $\|\cdot\|_{0}$. The standard $H^{1}(\Omega)$ seminorm will be denoted by $|\cdot|_{1}$. The norm in $L_{p}(\omega)$ is written as $\|\cdot\|_{L_{p}(\omega)}$ for general $p$. The measure of a subset $\omega$ of $\Omega$ is written $|\omega|$.

\section{A PRIORI BOUNDS ON THE SOLUTION $u$}

The papers [5, 6] consider (1.1) in the case of positive constants $p$ and $q$, with arbitrary data compatibility at each corner, and give a decomposition of the solution with pointwise bounds on the derivatives of the terms from this decomposition. As no corresponding analysis exists in the literature for variable positive functions $p$ and $q$, we shall make the reasonable assumption that the results of [5, 6] are also valid for (1.1). That is, we assume that

$$
u=v+E+z_{00}+z_{01}+z_{10}+z_{11},
$$

where for non-negative integers $m$ and $n$ and $(x, y) \in \Omega$ the derivatives of the various components satisfy the bounds 


$$
\begin{aligned}
&\left|D_{x}^{m} D_{y}^{n} v(x, y)\right| \leq C \\
&\left|D_{x}^{m} D_{y}^{n} E(x, y)\right| \leq C \varepsilon^{-m} e^{-\beta x / \varepsilon} \\
&\left|D_{x}^{m} D_{y}^{n} z_{1 \mu}(x, y)\right| \leq C\left[\varepsilon^{-n / 2}+\varepsilon^{1-m-n}\right] \text { for } m+n<2 \text { and } r_{1 \mu}<\varepsilon \\
&\left|D_{x}^{m} D_{y}^{n} z_{1 \mu}(x, y)\right| \leq C\left[\varepsilon^{-n / 2}+\varepsilon^{-1}\left|\ln r_{1 \mu}\right|\right] \text { for } m+n=2 \text { and } r_{1 \mu}<\varepsilon \\
&\left|D_{x}^{m} D_{y}^{n} z_{1 \mu}(x, y)\right| \leq C\left[\varepsilon^{-n / 2}+\varepsilon^{-1} r_{1 \mu}^{2-m-n}\right] \text { for } m+n>2 \text { and } r_{1 \mu}<\varepsilon \\
&\left|D_{x}^{m} D_{y}^{n} z_{10}(x, y)\right| \leq C \varepsilon^{-n / 2}\left[1+r_{10}^{1-m-n / 2}\right] e^{-\rho y / \sqrt{\varepsilon}} \text { for } \varepsilon \leq r_{10} \\
&\left|D_{x}^{m} D_{y}^{n} z_{11}(x, y)\right| \leq C \varepsilon^{-n / 2}\left[1+r_{11}^{1-m-n / 2}\right] e^{-\rho(1-y) / \sqrt{\varepsilon}} \text { for } \varepsilon \leq r_{11} \\
&\left|D_{x}^{m} D_{y}^{n} z_{0 \mu}(x, y)\right| \leq C\left[\varepsilon^{-m-n / 2}+\varepsilon^{1-m-n}\right] \text { for } m+n<2 \text { and } r_{0 \mu}<\varepsilon \\
&\left|D_{x}^{m} D_{y}^{n} z_{0 \mu}(x, y)\right| \leq C\left[\varepsilon^{-m-n / 2}+\varepsilon^{-1}\left|\ln r_{0 \mu}\right|\right] \text { for } m+n=2 \text { and } r_{0 \mu}<\varepsilon \\
&\left|D_{x}^{m} D_{y}^{n} z_{0 \mu}(x, y)\right| \leq C\left[\varepsilon^{-m-n / 2}+\varepsilon^{-1} r_{0 \mu}^{2-m-n}\right] \text { for } m+n>2 \text { and } r_{0 \mu}<\varepsilon \\
&\left|D_{x}^{m} D_{y}^{n} z_{00}(x, y)\right| \leq C \varepsilon^{-m-n / 2}\left[1+r_{00}^{1-n / 2}\right] e^{-\gamma y / \sqrt{\varepsilon}} e^{-\beta x / \varepsilon} \text { for } \varepsilon \leq r_{00}, \\
&\left|D_{x}^{m} D_{y}^{n} z_{01}(x, y)\right| \leq C \varepsilon^{-m-n / 2}\left[1+r_{01}^{1-n / 2}\right] e^{-\gamma(1-y) / \sqrt{\varepsilon}} e^{-\beta x / \varepsilon} \text { for } \varepsilon \leq r_{01}
\end{aligned}
$$

In these formulas $\mu \in\{0,1\}$ and $r_{i j}$ denotes the Euclidean distance from the point $(x, y)$ to the corner $(i, j)$, while $\gamma$ and $\rho$ are constants in the interval $\left(0, \sqrt{\min _{\bar{\Omega}} q} / 2\right)$. (In the case of constant coefficients the analysis of [5, 6] shows that one can take $\gamma=\rho=\sqrt{q} / 2$.)

Here $v$ is a smooth component of the solution, $E$ is the outflow boundary layer, and each $z_{i j}$ includes a corner singularity at the corner $(i, j)$; furthermore, as (2.2f) and (2.2g) show, the terms $z_{1 j}$ also contain parabolic boundary layers.

In our later analysis, a derivative such as $D_{x}^{2} D_{y} z_{01}$ is usually written as $z_{01, x x y}$.

\section{The SHISHKIn Mesh}

The tensor product Shishkin mesh that we shall construct on $\bar{\Omega}$ is the same as in 3, 4, it is piecewise equidistant in each coordinate direction, with a fine mesh at the outflow boundary $x=0$ and along the characteristic boundaries $y=0$ and $y=1$. (For a discussion of Shishkin meshes see [10, 11.) Our finite element method on this mesh will use globally continuous piecewise bilinears. Thus in Section 4.1 some local interpolation bounds are given for bilinears on anisotropic mesh rectangles.

Let $N$ be a positive integer that is divisible by 4 . Our tensor-product rectangular meshes will have $N$ intervals in each coordinate direction. Define the mesh transition points by

$$
\lambda_{x}=\min \left\{\frac{1}{2}, \frac{5}{2 \beta} \varepsilon \ln N\right\} \quad \text { and } \quad \lambda_{y}=\min \left\{\frac{1}{4}, \frac{2}{\min \{\gamma, \rho\}} \sqrt{\varepsilon} \ln N\right\} .
$$

The choice of $\lambda_{x}$ here is the same as in [3, 13. Assume that $\varepsilon$ is so small that (3.1) can be replaced by

$$
\lambda_{x}=\frac{5}{2 \beta} \varepsilon \ln N \quad \text { and } \quad \lambda_{y}=\frac{2}{\min \{\gamma, \rho\}} \sqrt{\varepsilon} \ln N,
$$




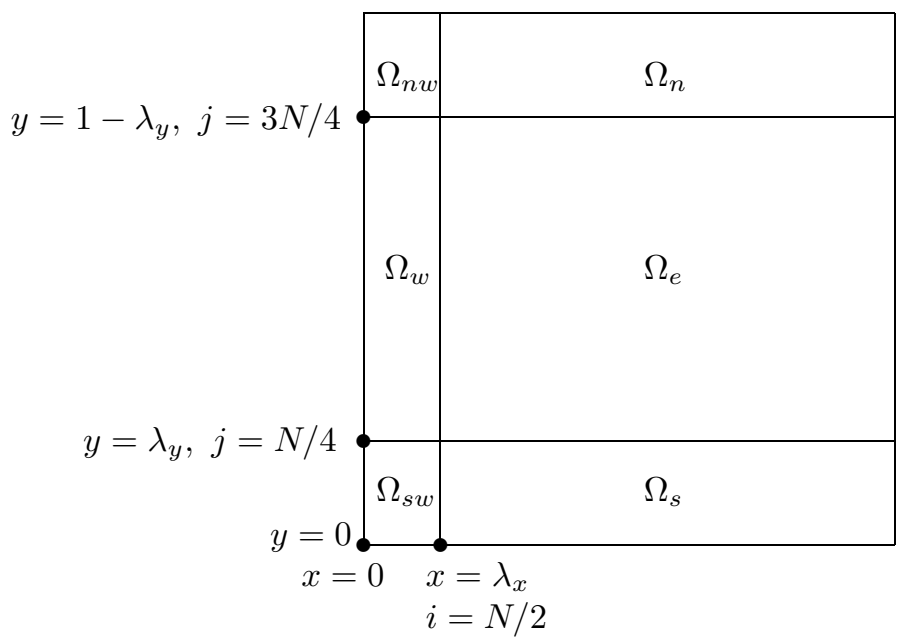

Figure 1. Decomposition of $\Omega$

as otherwise $N^{-1}$ is much smaller than $\varepsilon$ and the problem can be analysed in a classical manner. In fact for our analysis of the Galerkin method one could take $\lambda_{x}=(2 / \beta) \varepsilon \ln N$, but the analysis of the streamline diffusion method requires (3.2).

Set

$$
\begin{aligned}
& x_{i}=\frac{2 i \lambda_{x}}{N} \text { for } i=0, \ldots, \frac{1}{2} N, \\
& x_{i}=\lambda_{x}+\frac{2}{N}\left(1-\lambda_{x}\right)\left(i-\frac{N}{2}\right) \text { for } i=\frac{1}{2} N, \ldots, N, \\
& y_{j}=\lambda_{y}(4 j / N) \text { for } j=0, \ldots, \frac{1}{4} N, \\
& y_{j}=\lambda_{y}+\frac{2}{N}\left(1-2 \lambda_{y}\right)\left(j-\frac{1}{4} N\right) \text { for } j=\frac{1}{4} N, \ldots, \frac{3}{4} N, \\
& y_{j}=1-\lambda_{y}\left(4 N^{-1}(N-j)\right) \text { for } j=\frac{3}{4} N, \ldots, N .
\end{aligned}
$$

Set $h_{i}=x_{i}-x_{i-1}$ and $k_{j}=y_{j}-y_{j-1}$ for $i, j=1, \ldots, N$. Then the $h_{i}$ and $k_{j}$ satisfy the following bounds:

$$
\begin{aligned}
& h_{i}=2 \lambda_{x} N^{-1} \text { for } i=1,2, \ldots, \frac{1}{2} N, \\
& h_{i} \leq 2 N^{-1} \text { for } i=\frac{1}{2} N+1, \ldots, N, \\
& k_{j}=4 \lambda_{y} N^{-1} \text { for } j=1,2, \ldots, \frac{1}{4} N \text { and } j=\frac{3}{4} N+1, \ldots, N, \\
& k_{j} \leq 2 N^{-1} \text { for } j=\frac{1}{4} N+1, \ldots, \frac{3}{4} N .
\end{aligned}
$$

Our mesh corresponds to the decomposition of $\Omega$ displayed in Figure1, For later convenience, in the notation of Figure 1 1 set

$$
\Omega^{w}=\Omega_{s w} \cup \Omega_{w} \cup \Omega_{n w}, \quad \Omega^{s}=\Omega_{s w} \cup \Omega_{s} \quad \text { and } \quad \Omega^{n}=\Omega_{n w} \cup \Omega_{n} .
$$


3.1. Inequalities for $\epsilon$ and $N^{-1}$. Throughout the paper we make the reasonable practical assumption that

$$
\varepsilon \leq N^{-1}
$$

as otherwise it is straightforward to compute an accurate solution to (1.1).

Elementary calculus shows easily that

$$
N^{-1 / 2}(\ln N)^{3} \leq\left(\frac{6}{e}\right)^{3} \approx 10.75 \text { for } N \geq 4 .
$$

We appeal to this bound at several places in our analysis.

Likewise, for constant $r>0$, the function $x \mapsto x^{r}|\ln x|$ is increasing on the interval $0<x \leq 1 / e$; but $N^{-1}<1 / e$ since $N \geq 4$. Consequently,

$$
\varepsilon^{r}|\ln \varepsilon| \leq N^{-r}(\ln N) \quad \text { for } \varepsilon \leq N^{-1},
$$

which we use occasionally.

Remark 3.1. As the constants $C$ in our error estimates are of moderate size and independent of $\varepsilon$, the Shishkin mesh finite element method and its analysis are robust, even for problems that are severely singularly perturbed. Bounds such as (3.7) in this analysis would be much smaller if the $\ln N$ factor were absent but to remove such logarithmic factors would require the use of a more complicated mesh (e.g., a Bakhvalov mesh), and the analysis of our method would then become longer and more difficult.

\section{ERRORS IN INTERPOLATING TO $u$}

Let $V^{N} \subset C(\bar{\Omega})$ denote the conforming space of continuous piecewise bilinears on our Shishkin mesh. Given any function $w \in C(\bar{\Omega})$, we write $w^{I}$ for its nodal interpolant from $V^{N}$.

Define the energy norm $\||| w \mid\|_{\varepsilon}:=\left[\varepsilon|w|_{1}^{2}+\|w\|_{0}^{2}\right]^{1 / 2}$ for all $w \in H^{1}(\Omega)$. We shall estimate $u-u^{I}$ in both the $L_{2}(\Omega)$ and energy norms.

4.1. Interpolation inequalities for bilinears. The bounds in this section, which are quite general in their applicability, hold true on our mesh. Let $h_{i}$ and $k_{j}$ be as in the previous subsection. For each $i$ and $j$, let $\tau_{i j}$ denote the mesh rectangle $\left[x_{i-1}, x_{i}\right] \times\left[y_{j-1}, y_{j}\right]$. Then (see [1]) one has the following anisotropic interpolation inequalities for the bilinear nodal interpolant $w^{I}$ on $\tau_{i j}$ :

$$
\begin{aligned}
\left\|w-w^{I}\right\|_{L_{\infty}\left(\tau_{i j}\right)} & \leq C\|w\|_{L_{\infty}\left(\tau_{i j}\right)}, \\
\left\|w-w^{I}\right\|_{0, \tau_{i j}} & \leq C\left(h_{i}\left\|w_{x}\right\|_{0, \tau_{i j}}+k_{j}\left\|w_{y}\right\|_{0, \tau_{i j}}\right), \\
\left\|w-w^{I}\right\|_{0, \tau_{i j}} & \leq C\left(h_{i}^{2}\left\|w_{x x}\right\|_{0, \tau_{i j}}+k_{j}^{2}\left\|w_{y y}\right\|_{0, \tau_{i j}}\right), \\
\left\|\left(w-w^{I}\right)_{x}\right\|_{0, \tau_{i j}} & \leq C\left(h_{i}\left\|w_{x x}\right\|_{0, \tau_{i j}}+k_{j}\left\|w_{x y}\right\|_{0, \tau_{i j}}\right), \\
\left\|\left(w-w^{I}\right)_{x}\right\|_{L_{\infty}\left(\tau_{i j}\right)} & \leq 2\left\|w_{x}\right\|_{L_{\infty}\left(\tau_{i j}\right)} .
\end{aligned}
$$

There are analogous inequalities for $w_{y}$, which we shall also use. 


\subsection{The interpolation error estimate.}

Lemma 4.1. There exists a constant $C$ such that the $L_{2}$-norm interpolation error satisfies

$$
\left\|u-u^{I}\right\|_{0, \Omega} \leq C N^{-2} \ln N .
$$

Proof. Recall the decomposition (2.1): $u=v+E+z_{00}+z_{01}+z_{10}+z_{11}$. Consider first the smooth component $v$. By (4.1c) and (2.2a) one has

$$
\begin{aligned}
\left\|v-v^{I}\right\|_{0, \Omega}^{2} & \leq C \sum_{\tau_{i j} \subset \Omega}\left[h_{i}^{4}\left\|v_{x x}\right\|_{0, \tau_{i j}}^{2}+k_{j}^{4}\left\|v_{y y}\right\|_{0, \tau_{i j}}^{2}\right] \\
& \leq C N^{-4}\left(\left\|v_{x x}\right\|_{0, \Omega}^{2}+\left\|v_{y y}\right\|_{0, \Omega}^{2}\right) \leq C N^{-4} .
\end{aligned}
$$

For the exponential layer component $E$, outside $\Omega^{w}$ the stability bound (4.1a) and (2.2b) give

$$
\left\|E-E^{I}\right\|_{0, \Omega \backslash \Omega^{w}} \leq\left\|E-E^{I}\right\|_{L_{\infty}\left(\Omega \backslash \Omega^{w}\right)} \leq C\|E\|_{L_{\infty}\left(\Omega \backslash \Omega^{w}\right)} \leq C N^{-2},
$$

while on $\Omega^{w}$, by (4.1c) and (2.2b) we have

$$
\begin{aligned}
\left\|E-E^{I}\right\|_{0, \Omega^{w}}^{2} & \leq C \sum_{\tau_{i j} \subset \Omega^{w}}\left[h_{i}^{4}\left\|E_{x x}\right\|_{0, \tau_{i j}}^{2}+k_{j}^{4}\left\|E_{y y}\right\|_{0, \tau_{i j}}^{2}\right] \\
& \leq C \sum_{\tau_{i j} \subset \Omega^{w}}\left[h_{i}^{4} \iint_{\tau_{i j}} \varepsilon^{-4} e^{-2 \beta x / \varepsilon}+k_{j}^{4} \iint_{\tau_{i j}} e^{-2 \beta x / \varepsilon}\right] \\
& \leq C\left(N^{-1} \ln N\right)^{4} \iint_{\Omega^{w}} e^{-2 \beta x / \varepsilon} \leq C \varepsilon N^{-4}(\ln N)^{4} \leq C N^{-4}(\ln N)^{2},
\end{aligned}
$$

where we used (3.6) and (3.7). Hence

$$
\left\|E-E^{I}\right\|_{0} \leq C N^{-2} \ln N .
$$

Next, consider $z_{00}$. First, the stability result (4.1a) gives

$$
\left\|z_{00}-z_{00}^{I}\right\|_{0, \Omega \backslash \Omega_{s w}} \leq C\left\|z_{00}\right\|_{L_{\infty}\left(\Omega \backslash \Omega_{s w}\right)} \leq C N^{-2} \text {. }
$$

On $\Omega_{s w}$, by (4.1c) one has $\left\|z_{00}-z_{00}^{I}\right\|_{0, \Omega_{s w}}^{2} \leq C \sum_{\tau_{i j} \subset \Omega_{s w}} \iint_{\tau_{i j}}\left[h_{i}^{4} z_{00, x x}^{2}+k_{j}^{4} z_{00, y y}^{2}\right]$. Now (2.2i) and (2.2k) imply that

$$
\left|z_{00, x x}(x, y)\right| \leq C\left(\varepsilon^{-2}+\varepsilon^{-1}\left|\ln r_{00}\right|\right) \quad \text { and } \quad\left|z_{00, y y}(x, y)\right| \leq C \varepsilon^{-1}\left(1+\left|\ln r_{00}\right|\right)
$$

for all $(\xi, y) \in \Omega$. Consequently,

$$
\begin{aligned}
\left\|z_{00}-z_{00}^{I}\right\|_{0, \Omega_{s w}}^{2} & \leq C\left(N^{-1} \ln N\right)^{4} \iint_{\Omega_{s w}}\left[\varepsilon^{4}\left(\varepsilon^{-4}+\varepsilon^{-2}\left|\ln r_{00}\right|^{2}\right)\right. \\
& \left.+\varepsilon^{2} \varepsilon^{-2}\left(1+\left|\ln r_{00}\right|^{2}\right)\right] \\
& \leq C\left(N^{-1} \ln N\right)^{4} \iint_{\Omega_{s w}}\left(1+\left|\ln r_{00}\right|^{2}\right) \\
& \leq C\left(N^{-1} \ln N\right)^{4}\left[\left|\Omega_{s w}\right|+\int_{0}^{C \sqrt{\varepsilon} \ln N}\left|\ln r_{00}\right|^{2} r_{00} \mathrm{~d} r_{00}\right] \\
& \leq C\left(N^{-1} \ln N\right)^{4}\left[\varepsilon^{3 / 2}(\ln N)^{2}+\varepsilon(\ln N)^{2}|\ln (C \sqrt{\varepsilon} \ln N)|^{2}\right] \\
& \leq C \varepsilon^{1 / 2} N^{-4}(\ln N)^{5} \\
& \leq C N^{-4}(\ln N)^{2}
\end{aligned}
$$


where we used (3.6) and (3.7).

Combining (4.5) and (4.6) yields

$$
\left\|z_{00}-z_{00}^{I}\right\|_{0, \Omega} \leq C N^{-2} \ln N \text {. }
$$

Similarly, one obtains

$$
\left\|z_{01}-z_{01}^{I}\right\|_{0, \Omega} \leq C N^{-2} \ln N .
$$

We now come to the terms associated with the inflow corner singularities and parabolic layers. Consider $z_{10}$. First, on $\Omega \backslash \Omega^{s}$ one has

$$
\left\|z_{10}^{I}\right\|_{L^{\infty}\left(\Omega \backslash \Omega^{s}\right)} \leq\left\|z_{10}\right\|_{L^{\infty}\left(\Omega \backslash \Omega^{s}\right)} \leq C e^{-\rho \lambda_{y} / \sqrt{\varepsilon}} \leq C N^{-2},
$$

$\mathrm{SO}$

$$
\left\|z_{10}-z_{10}^{I}\right\|_{0, \Omega \backslash \Omega^{s}} \leq C N^{-2} .
$$

Let $R_{10}^{y}:=\bigcup_{j=1}^{N / 4} \tau_{N j}$ denote the rightmost column of mesh rectangles in $\Omega^{s}$. On $\Omega^{s} \backslash R_{10}^{y}$ one has $r_{10} \geq N^{-1} \geq \varepsilon$ by (3.6). Hence, by (4.1c) and (2.2f) one gets

$$
\begin{aligned}
\left\|z_{10}-z_{10}^{I}\right\|_{0, \Omega^{s} \backslash R_{10}^{y}}^{2} \leq & C \sum_{\tau_{i j} \subset \Omega^{s} \backslash R_{10}^{y}}\left[h_{i}^{4}\left\|z_{10, x x}\right\|_{0, \tau_{i j}}^{2}+k_{j}^{4}\left\|z_{10, y y}\right\|_{0, \tau_{i j}}^{2}\right] \\
\leq & C N^{-4} \iint_{\Omega^{s} \backslash R_{10}^{y}} r_{10}^{-2} r_{10} \mathrm{~d} r_{10} \mathrm{~d} \theta \\
& \quad+C \varepsilon^{2}\left(N^{-1} \ln N\right)^{4} \iint_{\Omega^{s} \backslash R_{10}^{y}} \varepsilon^{-2} e^{-2 \rho y / \sqrt{\varepsilon}} \\
& \leq C N^{-4} \ln N+C \varepsilon^{1 / 2}\left(N^{-1} \ln N\right)^{4} \\
& \leq C N^{-4} \ln N
\end{aligned}
$$

using $r_{10} \geq N^{-1}$ on $\Omega^{s} \backslash R_{10}^{y}$, (3.6) and (3.7).

It remains to estimate $\left\|z_{10}-z_{10}^{I}\right\|_{0, R_{10}^{y}}$. Let $D_{10}^{\varepsilon} \subset \Omega$ denote the quarter disk with centre $(1,0)$ and radius $\varepsilon$. Assume for the moment that $\varepsilon \leq N^{-3}$. Now $\left|R_{10}^{y}\right| \leq C N^{-1} \varepsilon^{1 / 2} \ln N$, so (4.1b), (2.2c) and (2.2f) yield

$$
\begin{aligned}
\left\|z_{10}-z_{10}^{I}\right\|_{0, R_{10}^{y}}^{2} \leq C \sum_{\tau_{i j} \subset R_{10}^{y}}\left[h_{i}^{2}\left\|z_{10, x}\right\|_{0, \tau_{i j}}^{2}+k_{j}^{2}\left\|z_{10, y}\right\|_{0, \tau_{i j}}^{2}\right] \\
\leq C N^{-2}\left(\iint_{D_{10}^{\varepsilon}}+\iint_{R_{10}^{y}} e^{-2 \rho y / \sqrt{\varepsilon}}\right) \\
\quad+C \varepsilon\left(N^{-1} \ln N\right)^{2}\left(\iint_{D_{10}^{\varepsilon}} \varepsilon^{-1}+\iint_{R_{10}^{y}} \varepsilon^{-1} e^{-2 \rho y / \sqrt{\varepsilon}}\right) \\
\leq C N^{-2}(\ln N)^{2}\left[\left|D_{10}^{\varepsilon}\right|+\left|R_{10}^{y}\right|\right] \leq C N^{-4},
\end{aligned}
$$

where we used $\varepsilon \leq N^{-3}$ and (3.7).

If instead $\varepsilon>N^{-3}$, then split $R_{10}^{y}$ as

$$
R_{10}^{y}=\overbrace{\left(\bigcup_{\tau_{i j} \cap D_{10}^{\varepsilon} \neq \emptyset} \tau_{i j}\right)}^{=: R_{10, l}^{y}} \cup \overbrace{\left(\bigcup_{\tau_{i j} \cap D_{10}^{\varepsilon}=\emptyset} \tau_{i j}\right)}^{=: R_{10, u}^{y}} .
$$


Then

$$
\begin{aligned}
\left\|z_{10}-z_{10}^{I}\right\|_{0, R_{10}^{y}}^{2} \leq C & \sum_{\tau_{i j} \subset R_{10, l}^{y}}\left[h_{i}^{2}\left\|z_{10, x}\right\|_{0, \tau_{i j}}^{2}+k_{j}^{2}\left\|z_{10, y}\right\|_{0, \tau_{i j}}^{2}\right] \\
& +C \sum_{\tau_{i j} \subset R_{10, u}^{y}}\left[h_{i}^{4}\left\|z_{10, x x}\right\|_{0, \tau_{i j}}^{2}+k_{j}^{4}\left\|z_{10, y y}\right\|_{0, \tau_{i j}}^{2}\right]
\end{aligned}
$$

by (4.1b) and (4.1c). For each $\tau_{i j} \subset R_{10}^{y}$ one has

$$
k_{j} \leq C \lambda_{y} N^{-1} \leq C \varepsilon^{1 / 2} N^{-1} \ln N \leq C N^{-3 / 2} \ln N
$$

by (3.6); consequently, the rectangle $R_{10, l}^{y}$ has height at most $\varepsilon+C N^{-3 / 2} \ln N \leq$ $C N^{-1}$. It follows that $\left|R_{10, l}^{y}\right| \leq C N^{-2}$. Thus (2.2c) and (2.2f) yield

$$
\begin{gathered}
\sum_{\tau_{i j} \subset R_{10, l}^{y}}\left[h_{i}^{2}\left\|z_{10, x}\right\|_{0, \tau_{i j}}^{2}+k_{j}^{2}\left\|z_{10, y}\right\|_{0, \tau_{i j}}^{2}\right] \\
\leq C N^{-2}\left(\iint_{D_{10}^{\varepsilon}}+\iint_{R_{10, l}^{y}} e^{-2 \rho y / \sqrt{\varepsilon}}\right) \\
+C \varepsilon\left(N^{-1} \ln N\right)^{2}\left(\iint_{D_{10}^{\varepsilon}} \varepsilon^{-1}+\iint_{R_{10, l}^{y}} \varepsilon^{-1} e^{-2 \rho y / \sqrt{\varepsilon}}\right) \\
\leq C N^{-2}(\ln N)^{2}\left[\left|D_{10}^{\varepsilon}\right|+\left|R_{10, l}^{y}\right|\right] \leq C N^{-4}(\ln N)^{2},
\end{gathered}
$$

where we used (3.6) again. Now (3.6) implies that $D_{10}^{\varepsilon} \subset R_{10, l}^{y}$, so $r_{10} \geq \varepsilon>N^{-3}$ on $\Omega \backslash R_{10, l}^{y}$. Then the bound (2.2f) yields

$$
\begin{gathered}
\sum_{\tau_{i j} \subset R_{10, u}^{y}}\left[h_{i}^{4}\left\|z_{10, x x}\right\|_{0, \tau_{i j}}^{2}+k_{j}^{4}\left\|z_{10, y y}\right\|_{0, \tau_{i j}}^{2}\right] \\
\leq C N^{-4} \iint_{R_{10, u}^{y}}\left(1+r_{10}^{-1}\right)^{2} e^{-2 \rho y / \sqrt{\varepsilon}} \\
\quad+C \varepsilon^{2}\left(N^{-1} \ln N\right)^{4} \iint_{R_{10, u}^{y}} \varepsilon^{-2} e^{-2 \rho y / \sqrt{\varepsilon}} \\
\leq C N^{-4} \iint_{R_{10, u}^{y}} r_{10}^{-1} d r_{10} d \theta+C \varepsilon^{1 / 2} N^{-5}(\ln N)^{4} \\
\leq C N^{-4} \ln N .
\end{gathered}
$$

From (4.9)-(4.14) we get

$$
\left\|z_{10}-z_{10}^{I}\right\|_{0, \Omega} \leq C N^{-2} \ln N .
$$

Analogously,

$$
\left\|z_{11}-z_{11}^{I}\right\|_{0, \Omega} \leq C N^{-2} \ln N .
$$

Combine (4.3), (4.4), (4.7), 4.8), (4.15) and (4.16) to complete the proof.

Lemma 4.2. There exists a constant $C$ such that the $\varepsilon$-weighted $H^{1}$-seminorm interpolation error satisfies

$$
\varepsilon^{1 / 2}\left\|\nabla\left(u-u^{I}\right)\right\|_{0, \Omega} \leq C N^{-1} \ln N .
$$


Proof. Again we rely on the decomposition $u=v+E+z_{00}+z_{01}+z_{10}+z_{11}$. Consider first the smooth component $v$, for which (4.1d) and (2.2a) yield

$$
\varepsilon\left\|\nabla\left(v-v^{I}\right)\right\|_{0, \Omega}^{2} \leq C \varepsilon \sum_{\tau_{i j} \subset \Omega}\left(h_{i}^{2}\left\|(\nabla v)_{x}\right\|_{0, \tau_{i j}}^{2}+k_{j}^{2}\left\|(\nabla v)_{y}\right\|_{0, \tau_{i j}}^{2}\right) \leq C \varepsilon N^{-2} .
$$

For the exponential layer component, apply triangle and inverse inequalities to obtain

$$
\begin{aligned}
\varepsilon\left\|\left(E-E^{I}\right)_{x}\right\|_{0, \Omega \backslash \Omega^{w}}^{2} & \leq C \varepsilon \sum_{i=N / 2+1}^{N} \sum_{j=1}^{N}\left(\left\|E_{x}\right\|_{0, \tau_{i j}}^{2}+\left\|E_{x}^{I}\right\|_{0, \tau_{i j}}^{2}\right) \\
& \leq C \varepsilon\left[\left\|E_{x}\right\|_{0, \Omega \backslash \Omega^{w}}^{2}+\sum_{i=N / 2+1}^{N} \sum_{j=1}^{N} h_{i}^{-2}\left|\tau_{i j}\right|\|E\|_{L_{\infty}\left(\tau_{i j}\right)}^{2}\right] \\
& \leq C\left[N^{-5}+\varepsilon N^{-5} \sum_{i=N / 2+1}^{N} \sum_{j=1}^{N} N k_{j}\right] \\
& \leq C\left(N^{-5}+\varepsilon N^{-3}\right) \\
& \leq C N^{-4}
\end{aligned}
$$

by (2.2b), (3.2) and (3.6). Inside $\Omega^{w}$ we apply (4.1d) and get

$$
\begin{aligned}
\varepsilon\left\|\left(E-E^{I}\right)_{x}\right\|_{0, \Omega^{w}}^{2} & \leq C \varepsilon \sum_{\tau_{i j} \subset \Omega^{w}}\left(h_{i}^{2}\left\|E_{x x}\right\|_{0, \tau_{i j}}^{2}+k_{j}^{2}\left\|E_{x y}\right\|_{0, \tau_{i j}}^{2}\right) \\
& \leq C \varepsilon^{-1}\left(N^{-1} \ln N\right)^{2}\left\|e^{-\beta x / \varepsilon}\right\|_{0, \Omega^{w}}^{2} \leq C\left(N^{-1} \ln N\right)^{2} .
\end{aligned}
$$

Thus

$$
\varepsilon^{1 / 2}\left\|\left(E-E^{I}\right)_{x}\right\|_{0, \Omega} \leq C N^{-1} \ln N .
$$

For $E_{y}$ apply (4.1e) outside $\Omega^{w}$ to obtain

$$
\varepsilon^{1 / 2}\left\|\left(E-E^{I}\right)_{y}\right\|_{0, \Omega \backslash \Omega^{w}} \leq C \varepsilon^{1 / 2}\left\|E_{y}\right\|_{L_{\infty}\left(\Omega \backslash \Omega^{w}\right)} \leq C \varepsilon^{1 / 2} N^{-5 / 2} \leq C N^{-3} .
$$

Inside $\Omega^{w}$ apply (4.1d), which gives

$$
\begin{aligned}
\varepsilon\left\|\left(E-E^{I}\right)_{y}\right\|_{0, \Omega^{w}}^{2} & \leq C \varepsilon \sum_{\tau_{i j} \subset \Omega^{w}}\left(h_{i}^{2}\left\|E_{x y}\right\|_{0, \tau_{i j}}^{2}+k_{j}^{2}\left\|E_{y y}\right\|_{0, \tau_{i j}}^{2}\right) \\
& \leq C \varepsilon\left(N^{-1} \ln N\right)^{2}\left\|e^{-\beta x / \varepsilon}\right\|_{0, \Omega^{w}}^{2} \\
& \leq C \varepsilon^{2}\left(N^{-1} \ln N\right)^{2} \leq C N^{-3}
\end{aligned}
$$

by (3.6). Altogether, we have shown that

$$
\varepsilon^{1 / 2}\left\|\left(E-E^{I}\right)_{y}\right\|_{0, \Omega} \leq C N^{-3 / 2} .
$$


We now tackle the outflow singularity $z_{00}$. The analysis of $z_{00}$ in $\Omega \backslash \Omega^{w}$ is similar to that for $E$ and via (2.2k) produces

$$
\begin{aligned}
\varepsilon\left\|\left(z_{00}-z_{00}^{I}\right)_{x}\right\|_{0, \Omega \backslash \Omega^{w}}^{2} & \leq \varepsilon \sum_{\tau_{i j} \in \Omega \backslash \Omega^{w}}\left[\left\|z_{00, x}\right\|_{0, \tau_{i j}}^{2}+\left|\tau_{i j}\right| h_{i}^{-2}\left\|z_{00}\right\|_{L_{\infty}\left(\tau_{i j}\right)}^{2}\right] \\
& \leq C \varepsilon\left(\varepsilon^{-2}\left\|e^{-\gamma y / \sqrt{\varepsilon}} e^{-\beta x / \varepsilon}\right\|_{0, \Omega \backslash \Omega^{w}}^{2}+N^{-5} \sum_{i=N / 2+1}^{N} N\right) \\
& \leq C\left(\varepsilon^{1 / 2} N^{-5}+\varepsilon N^{-3}\right) \leq C N^{-4}
\end{aligned}
$$

where we used (3.6). In $\Omega_{w} \cup \Omega_{n w}$ the stability bound (4.1e) and (2.2k) yield

$$
\begin{aligned}
\varepsilon^{1 / 2}\left\|\left(z_{00}-z_{00}^{I}\right)_{x}\right\|_{0, \Omega_{w} \cup \Omega_{n w}} & \leq C \varepsilon^{1 / 2}\left|\Omega_{w} \cup \Omega_{n w}\right|^{1 / 2}\left\|z_{00, x}\right\|_{L_{\infty}\left(\Omega_{w} \cup \Omega_{n w}\right)} \\
& \leq C \varepsilon^{1 / 2} \lambda_{x}^{1 / 2} \varepsilon^{-1}\left\|e^{-\gamma y / \sqrt{\varepsilon}} e^{-\beta x / \varepsilon}\right\|_{L_{\infty}\left(\Omega_{w} \cup \Omega_{n w}\right)} \\
& \leq C N^{-2}(\ln N)^{1 / 2} .
\end{aligned}
$$

Let $D_{00}^{\varepsilon}$ be the quarter-disc in $\Omega_{s w}$ with centre $(0,0)$ and radius $\varepsilon$. In $\Omega_{s w}$ apply (4.1d) with the bounds

$$
\begin{aligned}
& \left\|z_{00, x x}\right\|_{0, \tau_{i j}}^{2} \leq C\left(\iint_{\tau_{i j} \cap D_{00}^{\varepsilon}}\left(\varepsilon^{-4}+\varepsilon^{-2}\left|\ln r_{00}\right|^{2}\right)+\iint_{\tau_{i j} \backslash D_{00}^{\varepsilon}} \varepsilon^{-4} e^{-2 \gamma y / \sqrt{\varepsilon}} e^{-2 \beta x / \varepsilon}\right) \\
& \left\|z_{00, x y}\right\|_{0, \tau_{i j}}^{2} \leq C\left(\iint_{\tau_{i j} \cap D_{00}^{\varepsilon}}\left(\varepsilon^{-3}+\varepsilon^{-2}\left|\ln r_{00}\right|^{2}\right)+\iint_{\tau_{i j} \backslash D_{00}^{\varepsilon}} \varepsilon^{-3} e^{-2 \gamma y / \sqrt{\varepsilon}} e^{-2 \beta x / \varepsilon}\right),
\end{aligned}
$$

from (2.2i) and (2.2k). This leads to

$$
\begin{aligned}
\varepsilon\left\|\left(z_{00}-z_{00}^{I}\right)_{x}\right\|_{0, \Omega_{s w}}^{2} \leq C \varepsilon \sum_{\tau_{i j} \subset \Omega_{s w}}\left(h_{i}^{2}\left\|z_{00, x x}\right\|_{0, \tau_{i j}}^{2}+k_{j}^{2}\left\|z_{00, x y}\right\|_{0, \tau_{i j}}^{2}\right) \\
\leq C\left(N^{-1} \ln N\right)^{2}\left[\iint_{D_{00}^{\varepsilon}}\left(\varepsilon^{-1}+\left|\ln r_{00}\right|^{2}\right)\right. \\
\left.+\varepsilon^{-1} \iint_{\Omega_{s w} \backslash D_{00}^{\varepsilon}} e^{-2 \gamma y / \sqrt{\varepsilon}} e^{-2 \beta x / \varepsilon}\right] \\
\leq C \varepsilon^{1 / 2}\left(N^{-1} \ln N\right)^{2} \leq C N^{-5 / 2}(\ln N)^{2}
\end{aligned}
$$

on invoking (3.6). Combining (4.23)

$$
\varepsilon^{1 / 2}\left\|\left(z_{00}-z_{00}^{I}\right)_{x}\right\|_{0, \Omega} \leq C N^{-5 / 4} \ln N
$$

For $z_{00, y}$ on $\Omega \backslash \Omega^{w}$, if one proceeds similarly to the $x$-derivative the outcome is suboptimal because sums of $k_{j}^{-1}$ enter the calculation and contribute a factor $\varepsilon^{-1 / 2} N^{1}$; consequently, we start with the triangle inequality then avoid any inverse estimate by moving to the $L_{\infty}$ norm of $z_{00, y}$. Thus by (4.1e) and (2.2k) one gets

$$
\varepsilon\left\|\left(z_{00}-z_{00}^{I}\right)_{y}\right\|_{0, \Omega \backslash \Omega^{w}}^{2} \leq C \varepsilon\left\|z_{00, y}\right\|_{L_{\infty}\left(\Omega \backslash \Omega^{w}\right)}^{2} \leq C N^{-5}
$$


On $\Omega_{w} \cup \Omega_{n w}$ the stability bound (4.1e) and (2.2k) give

$$
\begin{aligned}
\varepsilon^{1 / 2}\left\|\left(z_{00}-z_{00}^{I}\right)_{y}\right\|_{0, \Omega_{w} \cup \Omega_{n w}} & \leq C \varepsilon^{1 / 2}\left|\Omega_{w} \cup \Omega_{n w}\right|^{1 / 2}\left\|z_{00, y}\right\|_{L_{\infty}\left(\Omega_{w} \cup \Omega_{n w}\right)} \\
& \leq C \varepsilon(\ln N)^{1 / 2} \varepsilon^{-1 / 2}\left\|e^{-\gamma y / \sqrt{\varepsilon}} e^{-\beta x / \varepsilon}\right\|_{L_{\infty}\left(\Omega_{w} \cup \Omega_{n w}\right)} \\
& \leq C N^{-5 / 2}(\ln N)^{1 / 2}
\end{aligned}
$$

by (3.6). On $\Omega_{s w}$, from (2.2il) one has

$$
\left\|z_{00, x y}\right\|_{0, \tau_{i j}}^{2} \leq C\left[\iint_{\tau_{i j} \cap D_{00}^{\varepsilon}}\left(\varepsilon^{-3}+\varepsilon^{-2}\left|\ln r_{00}\right|^{2}\right)+\iint_{\tau_{i j} \backslash D_{00}^{\varepsilon}} \varepsilon^{-3} e^{-2 \gamma y / \sqrt{\varepsilon}} e^{-2 \beta x / \varepsilon}\right]
$$

and

$$
\left\|z_{00, y y}\right\|_{0, \tau_{i j}}^{2} \leq C\left[\iint_{\tau_{i j} \cap D_{00}^{\varepsilon}}\left(\varepsilon^{-2}+\varepsilon^{-2}\left|\ln r_{00}\right|^{2}\right)+\iint_{\tau_{i j} \backslash D_{00}^{\varepsilon}} \varepsilon^{-2} e^{-2 \gamma y / \sqrt{\varepsilon}} e^{-2 \beta x / \varepsilon}\right] ;
$$

now apply (4.1d) to get

$$
\begin{aligned}
& \varepsilon\left\|\left(z_{00}-z_{00}^{I}\right)_{y}\right\|_{0, \Omega_{s w}}^{2} \leq C \varepsilon \sum_{\tau_{i j} \subset \Omega_{s w}}\left(h_{i}^{2}\left\|z_{00, x y}\right\|_{0, \tau_{i j}}^{2}+k_{j}^{2}\left\|z_{00, y y}\right\|_{0, \tau_{i j}}^{2}\right) \\
& \leq C\left(N^{-1} \ln N\right)^{2}\left[\iint_{D_{00}^{\varepsilon}}\left(1+\left|\ln r_{00}\right|^{2}\right)\right. \\
&\left.\quad+\iint_{\Omega_{s w} \backslash D_{00}^{\varepsilon}} e^{-2 \gamma y / \sqrt{\varepsilon}} e^{-2 \beta x / \varepsilon}\right] \\
& \leq C \varepsilon^{3 / 2}\left(N^{-1} \ln N\right)^{2} \leq C N^{-7 / 2}(\ln N)^{2}
\end{aligned}
$$

where (3.6) was also used. Combining (4.27)-(4.29), we have

$$
\varepsilon^{1 / 2}\left\|\left(z_{00}-z_{00}^{I}\right)_{y}\right\|_{0, \Omega} \leq C N^{-7 / 4} \ln N .
$$

One obtains analogous results for $z_{01}$.

Now consider the inflow singularity term $z_{10}$. We begin with its $y$ derivative. Outside $\Omega^{s}$ the stability result (4.1e) and (2.2f) yield

$$
\varepsilon^{1 / 2}\left\|\left(z_{10}-z_{10}^{I}\right)_{y}\right\|_{0, \Omega \backslash \Omega^{s}} \leq C \varepsilon^{1 / 2}\left\|z_{10, y}\right\|_{L_{\infty}\left(\Omega \backslash \Omega^{s}\right)} \leq C \varepsilon^{1 / 2} \varepsilon^{-1 / 2} N^{-2} \leq C N^{-2} .
$$

By (4.1d) one has

$$
\varepsilon\left\|\left(z_{10}-z_{10}^{I}\right)_{y}\right\|_{0, \Omega^{s}}^{2} \leq C \varepsilon \sum_{i=1}^{N} \sum_{j=1}^{N / 4}\left(h_{i}^{2}\left\|z_{10, x y}\right\|_{0, \tau_{i j}}^{2}+k_{j}^{2}\left\|z_{10, y y}\right\|_{0, \tau_{i j}}^{2}\right) .
$$

For each mesh rectangle $\tau_{i j}$, the bounds (2.2d) and (2.2f) provide the estimates

$$
\left\|z_{10, x y}\right\|_{0, \tau_{i j}}^{2} \leq C\left[\iint_{\tau_{i j} \cap D_{10}^{\varepsilon}}\left(\varepsilon^{-1}+\varepsilon^{-2}\left|\ln r_{10}\right|^{2}\right)+\iint_{\tau_{i j} \backslash D_{10}^{\varepsilon}} \varepsilon^{-1}\left(1+r_{10}^{-1}\right) e^{-2 \rho y / \sqrt{\varepsilon}}\right]
$$

and

$$
\left\|z_{10, y y}\right\|_{0, \tau_{i j}}^{2} \leq C\left[\iint_{\tau_{i j} \cap D_{10}^{\varepsilon}}\left(\varepsilon^{-2}+\varepsilon^{-2}\left|\ln r_{10}\right|^{2}\right)+\iint_{\tau_{i j} \backslash D_{10}^{\varepsilon}} \varepsilon^{-2} e^{-2 \rho y / \sqrt{\varepsilon}}\right] .
$$


Thus from (4.32) we obtain

$$
\begin{aligned}
& \varepsilon\left\|\left(z_{10}-z_{10}^{I}\right)_{y}\right\|_{0, \Omega^{s}}^{2} \\
& \leq C \varepsilon\left[N^{-2} \iint_{D_{10}^{\varepsilon}}\left(\varepsilon^{-1}+\varepsilon^{-2}\left|\ln r_{10}\right|^{2}\right)+N^{-2} \iint_{\Omega_{s} \backslash D_{10}^{\varepsilon}} \varepsilon^{-1} \frac{1}{r_{10}} r_{10} \mathrm{~d} r_{10} \mathrm{~d} \theta\right. \\
& \left.\quad+\left(N^{-1} \ln N\right)^{2} \iint_{D_{10}^{\varepsilon}} \varepsilon^{-1}\left(1+\left|\ln r_{10}\right|^{2}\right)+\left(N^{-1} \ln N\right)^{2} \iint_{\Omega_{s} \backslash D_{10}^{\varepsilon}} \varepsilon^{-1} e^{-2 \rho y / \sqrt{\varepsilon}}\right] \\
& \leq C\left[N^{-2}\left(\varepsilon^{2}+\varepsilon|\ln \varepsilon|^{2}\right)+N^{-2}+\left(N^{-1} \ln N\right)^{2}\left(\varepsilon^{2}+\varepsilon^{2}|\ln \varepsilon|^{2}\right)+\left(N^{-1} \ln N\right)^{2} \varepsilon^{1 / 2}\right] \\
& \leq C N^{-2}
\end{aligned}
$$

using (3.6). The bound on $z_{10, x x}$ is smaller than the bound on $z_{10, y y}$ and a similar argument yields

$$
\varepsilon\left\|\left(z_{10}-z_{10}^{I}\right)_{x}\right\|_{0, \Omega^{s}}^{2} \leq C N^{-2} .
$$

Thus we have shown that

$$
\varepsilon^{1 / 2}\left\|\nabla\left(z_{10}-z_{10}^{I}\right)\right\|_{0, \Omega} \leq C N^{-1} .
$$

A similar result is valid for $z_{11}$.

Combining (4.18), (4.20), (4.22), (4.26), (4.30) and (4.33), the proof is complete.

Theorem 4.3. There exists a constant $C$ such that the energy-norm interpolation error satisfies

$$
\left\|u-u^{I}\right\|_{\varepsilon} \leq C N^{-1} \ln N .
$$

Proof. This is immediate from Lemmas 4.1 and 4.2 .

\section{Galerkin ERROR ANALYSis FOR BILINEARS}

Define the bilinear form

$$
a_{\mathrm{Gal}}(v, w):=\varepsilon(\nabla v, \nabla w)-\left(p v_{x}, w\right)+(q v, w) \quad \forall v, w \in H_{0}^{1} .
$$

Assume without loss of generality that $q+(\operatorname{div} p) / 2 \geq C>0$ for some constant $C$, since a simple change of variable $u(x, y)=e^{k x} v(x, y)$ for some suitable constant $k$, combined with $p \geq \beta>0$ and $\varepsilon$ sufficiently small, will yield this standard inequality in finite element analyses. Then for some positive constant $C$ one has the coercivity inequality

$$
a_{\text {Gal }}(v, v) \geq C\left|\|v \mid\|_{\varepsilon}^{2} \quad \forall v \in H_{0}^{1} .\right.
$$

To solve (1.1) approximately, define the Galerkin piecewise bilinear finite element solution $u^{N} \in V^{N}$ by

$$
a_{\text {Gal }}\left(u^{N}, v^{N}\right)=\left(f, v^{N}\right) \text { for } v^{N} \in V^{N} .
$$

It follows easily that $u^{N}$ enjoys the Galerkin orthogonality property:

$$
a_{\mathrm{Gal}}\left(u-u^{N}, v^{N}\right)=0 \text { for } v^{N} \in V^{N} .
$$


Then by coercivity and Galerkin orthogonality one has

$$
\begin{aligned}
\left.C\left|\| u^{N}-u^{I}\right|\right|_{\varepsilon} ^{2} \leq a_{\mathrm{Gal}}\left(u^{N}-u^{I}, u^{N}-u^{I}\right)=a_{\mathrm{Gal}}\left(u-u^{I}, u^{N}-u^{I}\right) \\
\leq \varepsilon\left|\left(\nabla\left(u-u^{I}\right), \nabla\left(u^{N}-u^{I}\right)\right)\right|+\left|\left(p\left(u-u^{I}\right)_{x}, u^{N}-u^{I}\right)\right| \\
\quad+\left|\left(q\left(u-u^{I}\right), u^{N}-u^{I}\right)\right| \\
\leq\left. C N^{-1}(\ln N)||\left|u^{N}-u^{I}\right|\right|_{\varepsilon}+\left|\left(p\left(u-u^{I}\right)_{x}, u^{N}-u^{I}\right)\right|
\end{aligned}
$$

by Theorem 4.3 ,

Remark 5.1. By invoking an identity of Lin [7] (see [13] for its derivation), one can obtain the sharper estimate

$\varepsilon\left|\left(\nabla\left(u-u^{I}\right), \nabla\left(u^{N}-u^{I}\right)\right)\right|+\left|\left(q\left(u-u^{I}\right), u^{N}-u^{I}\right)\right| \leq C N^{-3 / 2}(\ln N)||\left|u^{N}-u^{I}\right| \|_{\varepsilon}$, but this improvement is useless here since later we can prove only the bound

$$
\left|\left(p\left(u-u^{I}\right)_{x}, u^{N}-u^{I}\right)\right| \leq\left. C N^{-1}(\ln N)\left|\| u^{N}-u^{I}\right|\right|_{\varepsilon}
$$

for the remaining term in (5.2).

Throughout the analysis that follows we consider $\left|\left(p\left(u-u^{I}\right)_{x}, \chi\right)\right|$ where $\chi \in V^{N}$ is arbitrary; eventually we shall take $\chi=u^{N}-u^{I}$.

5.1. The convection term. In considering the term $\left(p\left(u-u^{I}\right)_{x}, \chi\right)$ in (5.2), once again we use the decomposition (2.1).

Estimate of $\left(p\left(v-v^{I}\right)_{x}, \chi\right)$. This is very similar to the analysis of this term in [3, pp. 159, 160]. One obtains

$$
\left|\left(p\left(v-v^{I}\right)_{x}, \chi\right)\right| \leq C N^{-2}\|\chi\|_{0} .
$$

Estimate of $\left(p\left(E-E^{I}\right)_{x}, \chi\right)$. A minor variation of the argument used to handle this term in [3] yields

$$
\left|\left(p\left(E-E^{I}\right)_{x}, \chi\right)\right| \leq C N^{-2}(\ln N)^{2}\left|\|\chi \mid\|_{\varepsilon} .\right.
$$

Estimate of $\left(p\left(z_{00}-z_{00}^{I}\right)_{x}, \chi\right)$. Integrating by parts,

$$
\begin{aligned}
\left(p\left(z_{00}-z_{00}^{I}\right)_{x}, \chi\right)= & -\left(p_{x}\left(z_{00}-z_{00}^{I}\right), \chi\right) \\
& -\left(p\left(z_{00}-z_{00}^{I}\right), \chi_{x}\right)_{\Omega_{s w}}-\left(p\left(z_{00}-z_{00}^{I}\right), \chi_{x}\right)_{\Omega \backslash \Omega_{s w}} .
\end{aligned}
$$

Here

$$
\left|\left(p_{x}\left(z_{00}-z_{00}^{I}\right), \chi\right)\right| \leq C\left\|z_{00}-z_{00}^{I}\right\|_{0}\|\chi\|_{0} \leq C N^{-2}\left|\|\chi \mid\|_{\varepsilon}\right.
$$

by (4.7).

To deal with the next term in (5.5), we prove a modification of the bound on $\left\|z_{00}-z_{00}^{I}\right\|_{0, \Omega_{s w}}$ that was derived in the proof of Lemma 4.1. By (4.1b) one has $\left\|z_{00}-z_{00}^{I}\right\|_{0, \Omega_{s w}}^{2} \leq C \sum_{\tau_{i j} \subset \Omega_{s w}} \iint_{\tau_{i j}}\left[h_{i}^{2} z_{00, x}^{2}+k_{j}^{2} z_{00, y}^{2}\right]$. Now (2.2i) and (2.2k) imply that $\left|z_{00, x}(x, y)\right| \leq C \varepsilon^{-1}$ and $\left|z_{00, y}(x, y)\right| \leq C \varepsilon^{-1 / 2}$ for all $(\xi, y) \in \Omega$. Thus

$$
\begin{aligned}
\left\|z_{00}-z_{00}^{I}\right\|_{0, \Omega_{s w}}^{2} & \leq C\left(N^{-1} \ln N\right)^{2} \iint_{\Omega_{s w}}\left[\varepsilon^{4}\left(\varepsilon^{-2}\right)+\varepsilon\left(\varepsilon^{-1}\right)\right] \\
& \leq C\left(N^{-1} \ln N\right)^{2}\left|\Omega_{s w}\right|=C \varepsilon^{3 / 2} N^{-2}(\ln N)^{4}
\end{aligned}
$$


Consequently,

$$
\begin{aligned}
\left|\left(p\left(z_{00}-z_{00}^{I}\right), \chi_{x}\right)_{\Omega_{s w}}\right| \leq C\left\|z_{00}-z_{00}^{I}\right\|_{0, \Omega_{s w}}\left\|\chi_{x}\right\|_{0} & \leq C \varepsilon^{1 / 4} N^{-1}(\ln N)^{2} \mid\|\chi\| \|_{\varepsilon} \\
& \leq C N^{-1}(\ln N) \mid\|\chi\| \|_{\varepsilon}
\end{aligned}
$$

by (3.6) and (3.7).

To estimate $\left(p\left(z_{00}-z_{00}^{I}\right), \chi_{x}\right)_{\Omega \backslash \Omega_{s w}}$ write $\Omega \backslash \Omega_{s w}=A \cup B$ where

$$
A=\left(0, \lambda_{x}\right) \times\left(\lambda_{y}, 1\right) \text { and } B=\left(\lambda_{x}, 1\right) \times(0,1) .
$$

The inner product is estimated separately over each region. First, $\left\|z_{00}^{I}\right\|_{L_{\infty}(A)} \leq$ $\left\|z_{00}\right\|_{L_{\infty}(A)} \leq C e^{-\gamma \lambda_{y} / \sqrt{\varepsilon}}$ so

$$
\begin{aligned}
\left|\left(z_{00}-z_{00}^{I}, \chi_{x}\right)_{A}\right| & \leq C e^{-\gamma \lambda_{y} / \sqrt{\varepsilon}}|A|^{1 / 2}\left\|\chi_{x}\right\|_{0} \leq C N^{-2} \lambda_{x}^{1 / 2}\left\|\chi_{x}\right\|_{0} \\
& \leq C N^{-2}(\ln N)^{1 / 2}\|\chi\|_{\varepsilon} .
\end{aligned}
$$

Next, $\left\|z_{00}^{I}\right\|_{L_{\infty}(B)} \leq\left\|z_{00}\right\|_{L_{\infty}(B)} \leq C e^{-\beta \lambda_{x} / \varepsilon} \leq C N^{-5 / 2}$ so

$$
\left|\left(z_{00}-z_{00}^{I}, \chi_{x}\right)_{B}\right| \leq C N^{-5 / 2}|B|^{1 / 2}\left\|\chi_{x}\right\|_{0, B} \leq C N^{-3 / 2}\|\chi\|_{0, B} \leq C N^{-3 / 2}\||\chi|\|_{\varepsilon}
$$

by an inverse inequality. Collecting these inequalities yields

$$
\left|\left(p\left(z_{00}-z_{00}^{I}\right), \chi_{x}\right)_{\Omega \backslash \Omega_{s w}}\right| \leq C N^{-3 / 2}\left|\|\chi \mid\|_{\varepsilon} .\right.
$$

By virtue of (5.5)-(5.8) we obtain

$$
\left|\left(p\left(z_{00}-z_{00}^{I}\right)_{x}, \chi\right)\right| \leq C N^{-1}(\ln N)\left|\|\chi \mid\|_{\varepsilon} .\right.
$$

Estimate of $\left(p\left(z_{10}-z_{10}^{I}\right)_{x}, \chi\right)$. On $\Omega \backslash \Omega^{s}$, by (2.2f) one gets

$$
\left|z_{10, x}(x, y)\right| \leq C e^{-\rho \lambda_{y} / \sqrt{\varepsilon}} \leq C N^{-2},
$$

and it follows that $\left|z_{10, x}^{I}(x, y)\right| \leq C N^{-2}$ on $\Omega \backslash \Omega^{s}$ also. Hence

$$
\left\|\left(z_{10}-z_{10}^{I}\right)_{x}\right\|_{0, \Omega \backslash \Omega^{s}} \leq C N^{-2} .
$$

Recall from Section 4 that $D_{10}^{\varepsilon} \subset \Omega$ is the quarter disk with centre $(1,0)$ and radius $\varepsilon$, and $R_{10}^{y}=\bigcup_{j=1}^{N / 4} \tau_{N j}$ is the rightmost strip of mesh rectangles in $\Omega^{s}$; one has $D_{10}^{\varepsilon} \subset R_{10}^{y}$ since $\varepsilon \leq N^{-1}$, and $r_{10} \geq C N^{-1}$ on $\Omega^{s} \backslash R_{10}^{y}$. By (4.1d) and (2.2f) we have

$$
\begin{aligned}
\left\|\left(z_{10}-z_{10}^{I}\right)_{x}\right\|_{0, \Omega^{s} \backslash R_{10}^{y}}^{2} \leq C \sum_{\tau_{i j} \subset\left(\Omega^{s} \backslash R_{10}^{y}\right)} \iint_{\tau_{i j}}\left(h_{i}^{2} z_{10, x x}^{2}+k_{j}^{2} z_{10, x y}^{2}\right) \\
\leq C\left[N^{-2} \iint_{\Omega^{s} \backslash R_{10}^{y}} r_{10}^{-2} r_{10} \mathrm{~d} r_{10} \mathrm{~d} \theta\right. \\
\left.\quad+\varepsilon N^{-2}(\ln N)^{2} \iint_{\Omega^{s} \backslash R_{10}^{y}} \varepsilon^{-1} r_{10}^{-1} r_{10} \mathrm{~d} r_{10} \mathrm{~d} \theta\right] \\
\leq C\left[N^{-2} \ln N+N^{-2}(\ln N)^{2}\right] \\
\leq C N^{-2}(\ln N)^{2} .
\end{aligned}
$$

The inequalities (5.10) and (5.11) imply that

$$
\left|\left(p\left(z_{10}-z_{10}^{I}\right)_{x}, \chi\right)_{\Omega \backslash R_{10}^{y}}\right| \leq C\left\|\left(z_{10}-z_{10}^{I}\right)_{x}\right\|_{0, \Omega}\|\chi\|_{0, \Omega} \leq C N^{-1}(\ln N)\|\chi\|_{0} .
$$


Finally, as $\left\|z_{10, x}^{I}\right\|_{L_{\infty}(\Omega)} \leq\left\|z_{10, x}\right\|_{L_{\infty}(\Omega)} \leq C$, the Cauchy-Schwarz inequality yields

$$
\begin{aligned}
& \left|\left(p\left(z_{10}-z_{10}^{I}\right)_{x}, \chi\right)_{R_{10}^{y}}\right| \\
& \leq C\left\|\left(z_{10}-z_{10}^{I}\right)_{x}\right\|_{0, R_{10}^{y}}\|\chi\|_{0, R_{10}^{y}} \\
& \leq C\left|R_{10}^{y}\right|^{1 / 2}\|\chi\|_{0, R_{10}^{y}} \\
& \leq C \varepsilon^{1 / 4} N^{-1 / 2}(\ln N)^{1 / 2}\|\chi\|_{0, R_{10}^{y}}^{1 / 2}\|\chi\|_{0, R_{10}^{y}}^{1 / 2} \\
& \leq C \varepsilon^{1 / 4} N^{-1 / 2}(\ln N)^{1 / 2}\|\chi\|_{0, R_{10}^{y}}^{1 / 2}\left\{\int_{y=0}^{\lambda_{y}} \int_{x=x_{N-1}}^{1} h_{N} \int_{t=x_{N-1}}^{1}\left|\chi_{x}(t, y)\right|^{2} d t d x d y\right\}^{1 / 4},
\end{aligned}
$$

since $\chi(1, y)=0$ for all $y$ implies that for $(x, y) \in R_{10}^{y}$ one has

$$
|\chi(x, y)| \leq \int_{t=x}^{1}\left|\chi_{x}(t, y)\right| d y \leq h_{N}^{1 / 2}\left(\int_{t=x_{N-1}}^{1}\left|\chi_{x}(t, y)\right|^{2} d y\right)^{1 / 2}
$$

via a Cauchy-Schwarz inequality. Consequently,

$$
\begin{aligned}
\left|\left(p\left(z_{10}-z_{10}^{I}\right)_{x}, \chi\right)_{R_{10}^{y}}\right| & \leq C \varepsilon^{1 / 4} N^{-1}(\ln N)^{1 / 2}\|\chi\|_{0, R_{10}^{y}}^{1 / 2}\left\|\chi_{x}\right\|_{0, R_{10}^{y}}^{1 / 2} \\
& =C N^{-1}(\ln N)^{1 / 2}\|\chi\|_{0, R_{10}^{y}}^{1 / 2}\left(\varepsilon^{1 / 2}\left\|\chi_{x}\right\|_{0, R_{10}^{y}}\right)^{1 / 2} \\
& \leq C N^{-1}(\ln N)^{1 / 2}\|\| \chi \|_{\varepsilon}
\end{aligned}
$$

From (5.12) and (5.13) we have

$$
\left|\left(p\left(z_{10}-z_{10}^{I}\right)_{x}, \chi\right)\right| \leq C N^{-1}(\ln N)\left|\|\chi \mid\|_{\varepsilon} .\right.
$$

Then (5.3), (5.4), (5.9) and (5.14) (and analogous bounds for $z_{10}$ and $z_{11}$ ) yield

$$
\left|\left(p\left(u-u^{I}\right)_{x}, \chi\right)\right| \leq C N^{-1}(\ln N) \mid\|\chi\|_{\varepsilon} .
$$

Remark 5.2. The analysis of the diffusion terms in 3] draws on an integral identity of Lin [7, 13] to demonstrate the optimal order of convergence. There is a related but more complicated formula for convection terms (see [3. Lemma 4.3]) that when applied here would leave a negative power of $\varepsilon$ in the final bound (5.15), so we do not invoke it in our analysis.

Theorem 5.3. The Galerkin solution $u^{N}$ satisfies the error estimate

$$
\left\|u^{N}-u^{I} \mid\right\|_{\varepsilon} \leq C N^{-1} \ln N .
$$

Proof. Take $\chi=u^{N}-u^{I}$ in (5.15) then substitute this bound into (5.2).

Corollary 5.4. There exists a constant $C$ such that

$$
\|\left|u-u^{N}\right|||_{\varepsilon} \leq C N^{-1} \ln N .
$$

Proof. Combine Theorems 5.3 and 4.3

Comparing Corollary 5.4 with Theorem 4.3 shows that with respect to the energy norm \|\|$\cdot \|\left.\right|_{\varepsilon}$ the Galerkin solution $u^{N}$ achieves the same order of accuracy as the nodal interpolant $u^{I}$. 


\section{The Streamline diffusion finite ElEment Method}

Solutions of convection-diffusion problems that are computed by the standard Galerkin finite element method generally exhibit oscillations, even on Shishkin meshes, and moreover the associated discrete algebraic systems cannot be solved efficiently; see 9. To ameliorate these problems, stabilised finite element methods are generally applied to convection-diffusion problems. Several such methods are described in 10. One popular stabilised method is the streamline diffusion finite element method (SDFEM) which we now describe and analyse.

In the SDFEM one adds weighted residuals to the standard Galerkin method (5.1). Begin by defining the bilinear form

$$
a_{s t a b}(w, v):=\sum_{\tau \in T^{N}} \delta_{\tau}\left(\varepsilon \Delta w+p w_{x}-q w, p v_{x}\right)_{\tau}
$$

provided that $w \in H^{2}(\tau)$ and $v \in H^{1}(\tau)$ for all $\tau \in T^{N}$. Here the user-chosen parameters $\delta_{\tau} \geq 0$, which are constant on each $\tau$, will be specified later. Then the SDFEM is: Find $w^{N} \in V^{N}$ such that

$$
a_{S D}\left(w^{N}, v^{N}\right):=a_{G a l}\left(w^{N}, v^{N}\right)+a_{s t a b}\left(w^{N}, v^{N}\right)=f_{S D}\left(v^{N}\right) \quad \text { for all } v^{N} \in V^{N},
$$

with

$$
f_{S D}(u, v):=(f, v)-\sum_{\tau \in T^{N}} \delta_{\tau}\left(f, p v_{x}\right)_{\tau} .
$$

The SDFEM is consistent with (1.1), i.e., the solution $u$ of (1.1) satisfies $a_{S D}\left(u^{N}, v^{N}\right)=f_{S D}\left(v^{N}\right)$ for all $v^{N} \in V^{N}$. Furthermore, the SDFEM enjoys the Galerkin orthogonality property

$$
a_{S D}\left(u-w^{N}, v^{N}\right)=0 \quad \text { for all } v^{N} \in V^{N} .
$$

Define the streamline diffusion norm $\||\cdot|\|$ by

$$
\|v\|_{S D}^{2}:=\|v\|_{\varepsilon}^{2}+\sum_{\tau \in T^{N}} \delta_{\tau}\left(p v_{x}, p v_{x}\right)_{\tau} .
$$

Assume as in Section [5] that $q+(\operatorname{div} p) / 2 \geq C_{1}>0$ for some constant $C_{1}$. It is shown in, e.g., [10, III.3.2.1] that if $0 \leq \delta_{\tau}$ and $2 \delta_{\tau}\|q\|_{L_{\infty}(\tau)}^{2} \leq C_{1}$ for all $\tau \in T^{N}$, then one obtains coercivity with respect to $\|\mid\| \cdot \|_{S D}$ :

$$
a_{S D}(v, v) \geq \frac{1}{2}\|v\|_{S D}^{2} \quad \forall v \in V^{N} .
$$

The coercivity and Galerkin orthogonality properties imply that

$$
\frac{1}{2}\left\|u^{I}-w^{N}\right\|_{S D}^{2} \leq a_{G a l}\left(u^{I}-u, u^{I}-w^{N}\right)+a_{s t a b}\left(u^{I}-u, u^{I}-w^{N}\right) .
$$

Here, combining (5.15) with the calculation leading to (5.2), we have

$$
\left|a_{G a l}\left(u^{I}-u, \chi\right)\right| \leq C N^{-1}(\ln N)\|\| \chi \mid \|_{\varepsilon} \quad \text { for all } \chi \in V^{N} .
$$

Thus to bound $\left\|\mid u^{I}-w^{N}\right\| \|$ we must estimate the term $a_{\text {stab }}(\cdot, \cdot)$ in (6.3). While doing this we analyse the influence of the parameter $\delta_{\tau}$ on the stability and accuracy of the SDFEM. Although a term of the same form $a_{\text {stab }}\left(u^{I}-u, u^{I}-w^{N}\right)$ was bounded in [4, the solution $u$ of (1.1) behaves so differently from the function $u$ 
of [4] that very little of the analysis of [4] can be reused here and consequently, apart from Lemma 6.1, we do not quote any results from [4] below.

In our analysis we shall make the reasonable assumption that $\delta_{\tau}$ takes the constant value $\delta_{*}$ on each mesh region $\Omega_{*}$, where $\Omega_{*}$ is any one of the 6 mesh regions $\Omega_{w}, \Omega_{s w}$, etc., in Figure 1. Note that the mesh is uniform on each $\Omega_{*}$.

The reaction term in $a_{\text {stab }}\left(u^{I}-u, u^{I}-w^{N}\right)$ is easily handled: by Lemma 4.1 and the definition of $\||\cdot|\|_{S D}$ one has immediately the bound

$$
\delta_{*}\left|\left(q\left(u-u^{I}\right), p \chi_{x}\right)_{\Omega_{*}}\right| \leq C \delta_{*}^{1 / 2} N^{-2}(\ln N)\left|\|\chi \mid\|_{S D}\right.
$$

for each mesh region $\Omega_{*}$ and all $\chi \in V^{N}$.

We bound the diffusion and convection terms in $a_{\text {stab }}\left(u^{I}-u, u^{I}-w^{N}\right)$ by considering them on each separate mesh region in Sections 6.1 6.4. The diffusion term is simplified by the observation that, since $u^{I}$ is piecewise bilinear, one has $\Delta u^{I}=0$ on each mesh rectangle $\tau$. We again use the decomposition $u=v+E+z_{00}+z_{01}+z_{10}+z_{11}$ of (2.1).

The following two lemmas are used several times in the subsequent analysis.

Lemma 6.1. Let $p \in W_{\infty}^{1}(\Omega)$. Let $\Omega_{*}$ be any mesh region of our Shishkin mesh. Denote by $h_{*}$ and $k_{*}$ the meshsizes in the $x$ and $y$ directions inside $\Omega_{*}$. Let $\varphi \in$ $H^{2}\left(\Omega_{*}\right)$ and $\chi \in V^{N}$. Then

$$
\begin{aligned}
\left|\left(p\left(\varphi-\varphi^{I}\right)_{x}, p \chi_{x}\right)_{\Omega_{*}}\right| \leq C[ & \left(h_{*}+k_{*}\right)\left(h_{*}\left\|\varphi_{x x}\right\|_{0, \Omega_{*}}+k_{*}\left\|\varphi_{x y}\right\|_{0, \Omega_{*}}\right) \\
& \left.+k_{*}^{2}\left\|\varphi_{x y y}\right\|_{0, \Omega_{*}}\right]\left\|\chi_{x}\right\|_{0, \Omega_{*}},
\end{aligned}
$$

where $C$ is independent of $\varphi$ and $\chi$.

Proof. See [4, Proposition 8].

Lemma 6.2. Let $\tau$ be a mesh rectangle with base length $h$ and height $k$. Let $\chi \in V^{N}$. Then

$$
\begin{gathered}
\left\|\chi_{x}\right\|_{L_{p}(\tau)} \leq C h^{-1}\|\chi\|_{L_{p}(\tau)}, \quad\left\|\chi_{y}\right\|_{L_{p}(\tau)} \leq C k^{-1}\|\chi\|_{L_{p}(\tau)}, \\
\|\chi\|_{L_{q}(\tau)} \leq C(h k)^{1 / q-1 / p}\|\chi\|_{L_{p}(\tau)}
\end{gathered}
$$

for all $p, q \in[1, \infty]$, where the constant $C$ is independent of $\tau$ and $\chi$.

Proof. These finite element inverse estimates are well known.

The inequality $\left|\|\cdot\|\left\|_{\varepsilon} \leq\right\|\right| \cdot \mid \|_{S D}$ and the equivalence of $\left\|p \chi_{x}\right\|$ and $\left\|\chi_{x}\right\|$ for various norms $\|\cdot\|$ will be used frequently in the analysis. Throughout, $\chi \in V^{N}$ is arbitrary.

6.1. Subdomain $\Omega_{e}$. For $v$, by (2.2a wave

$$
\delta_{e} \varepsilon\left|\left(\Delta v, p \chi_{x}\right)_{\Omega_{e}}\right| \leq \delta_{e} \varepsilon\|\Delta v\|_{0, \Omega_{e}}\left\|\chi_{x}\right\|_{0, \Omega_{e}} \leq C \delta_{e} \varepsilon^{1 / 2}\|\chi\|_{\varepsilon}
$$

and, by Lemma 6.1

$$
\delta_{e}\left|\left(p\left(v-v^{I}\right)_{x}, p \chi_{x}\right)_{\Omega_{e}}\right| \leq C \delta_{e} N^{-2}\left\|\chi_{x}\right\|_{0, \Omega_{e}} \leq C \delta_{e}^{1 / 2} N^{-2}\|\chi\|_{S D} .
$$

Set $\eta_{1}=E+z_{00}+z_{01}$. By (2.2) and Lemma 6.2 we have

$$
\begin{aligned}
\delta_{e} \varepsilon\left|\left(\Delta \eta_{1}, p \chi_{x}\right)_{\Omega_{e}}\right| & \leq C \delta_{e} \varepsilon\left\|\Delta \eta_{1}\right\|_{L_{1}\left(\Omega_{e}\right)}\left\|\chi_{x}\right\|_{L_{\infty}\left(\Omega_{e}\right)} \leq C \delta_{e} N^{-3 / 2}\left\|\chi_{x}\right\|_{0, \Omega_{e}} \\
& \leq C \delta_{e}^{1 / 2} N^{-3 / 2}\|\| \|_{S D}
\end{aligned}
$$


and

$$
\begin{aligned}
\delta_{e}\left|\left(p\left(\eta_{1}-\eta_{1}^{I}\right)_{x}, p \chi_{x}\right)_{\Omega_{e}}\right| & \leq C \delta_{e}\left(\left\|\eta_{1, x}\right\|_{L_{1}\left(\Omega_{e}\right)} N\left\|\chi_{x}\right\|_{0, \Omega_{e}}+N\left\|\eta_{1}^{I}\right\|_{L_{\infty}\left(\Omega_{e}\right)}\left\|\chi_{x}\right\|_{0, \Omega_{e}}\right) \\
& \leq C \delta_{e}^{1 / 2} N^{-3 / 2}\|\| \chi \|_{S D} .
\end{aligned}
$$

For the inflow singularities, setting $\eta_{2}=z_{10}+z_{11}$ one has, similarly,

$$
\begin{aligned}
\delta_{e} \varepsilon\left|\left(\Delta \eta_{2}, p \chi_{x}\right)_{\Omega_{e}}\right| & \leq C \varepsilon \delta_{e}\left\|\Delta \eta_{2}\right\|_{L_{1}\left(\Omega_{e}\right)}\left\|\chi_{x}\right\|_{L_{\infty}\left(\Omega_{e}\right)} \leq C \varepsilon^{1 / 2} \delta_{e} N^{-1}\left\|\chi_{x}\right\|_{0, \Omega_{e}} \\
& \leq C \delta_{e} N^{-1}\|\chi\| \|_{S D}
\end{aligned}
$$

and

$$
\begin{aligned}
\delta_{e}\left|\left(p\left(\eta_{2}-\eta_{2}^{I}\right)_{x}, p \chi_{x}\right)_{\Omega_{e}}\right| & \leq C \delta_{e}\left\|\left(\eta_{2}-\eta_{2}^{I}\right)_{x}\right\|_{L_{\infty}\left(\Omega_{e}\right)}\left\|\chi_{x}\right\|_{0, \Omega_{e}} \\
& \leq C \delta_{e}\left\|\eta_{2, x}\right\|_{L_{\infty}\left(\Omega_{e}\right)}\left\|\chi_{x}\right\|_{0, \Omega_{e}} \leq C \delta_{e}^{1 / 2} N^{-2}\|\chi\|_{\varepsilon} .
\end{aligned}
$$

Combining these inequalities with (6.5), we have

$$
\left|a_{s t a b}\left(u-u^{I}, \chi\right)_{\Omega_{e}}\right| \leq C\left[\delta_{e}\left(\varepsilon^{1 / 2}+N^{-1}\right)+\delta_{e}^{1 / 2} N^{-3 / 2}\right] \mid\|\chi\|_{S D}
$$

for all $\chi \in V^{N}$.

6.2. Subdomain $\Omega_{w}$. Set $\eta_{3}=v+E$. Then by (2.2) we have

$$
\begin{aligned}
\delta_{w} \varepsilon\left|\left(\Delta \eta_{3}, p \chi_{x}\right)_{\Omega_{w}}\right| & \leq C \delta_{w} \varepsilon\left\|\Delta \eta_{3}\right\|_{0, \Omega_{w}}\left\|\chi_{x}\right\|_{0, \Omega_{w}} \leq C \delta_{w} \varepsilon^{-1 / 2}\left\|\chi_{x}\right\|_{0, \Omega_{w}} \\
& \leq C \delta_{w} \varepsilon^{-1}\|\chi\|_{\varepsilon},
\end{aligned}
$$

and, by Lemma 6.2

$$
\begin{aligned}
& \delta_{w}\left|\left(p\left(\eta_{3}-\eta_{3}^{I}\right)_{x}, p \chi_{x}\right)_{\Omega_{w}}\right| \\
& \quad \leq C \delta_{w} N^{-2}\left[\varepsilon(\ln N)\left\|\eta_{3, x x}\right\|_{0, \Omega_{w}}+\left\|\eta_{3, x y}\right\|_{0, \Omega_{w}}+\left\|\eta_{3, x y y}\right\|_{0, \Omega_{w}}\right]\left\|\chi_{x}\right\|_{0, \Omega_{w}} \\
& \quad \leq C \delta_{w} \varepsilon^{-1 / 2} N^{-2}(\ln N)\left\|\chi_{x}\right\|_{0, \Omega_{w}} \\
& \quad \leq C \delta_{w} \varepsilon^{-1} N^{-2}(\ln N)\|\chi\| \|_{\varepsilon} .
\end{aligned}
$$

Set $\eta_{4}=z_{00}+z_{01}+z_{10}+z_{11}$. Then from (2.2) and Lemma 6.2 one gets

$$
\begin{aligned}
\delta_{w} \varepsilon\left|\left(\Delta \eta_{4}, p \chi_{x}\right)_{\Omega_{w}}\right| & \leq C \delta_{w} \varepsilon\left\|\Delta \eta_{4}\right\|_{L_{1}\left(\Omega_{w}\right)}\left\|\chi_{x}\right\|_{L_{\infty}\left(\Omega_{w}\right)} \\
& \leq C \delta_{w} \varepsilon^{1 / 2} N^{-2}\left(\varepsilon N^{-2} \ln N\right)^{-1 / 2}\left\|\chi_{x}\right\|_{0, \Omega_{w}} \\
& \leq C \delta_{w} \varepsilon^{-1 / 2} N^{-1}(\ln N)^{-1 / 2}\|\chi\|_{\varepsilon}
\end{aligned}
$$

and, by Lemma 4.2 applied in the case where $v+E \equiv 0$,

$$
\begin{aligned}
\delta_{w}\left|\left(p\left(\eta_{4}-\eta_{4}^{I}\right)_{x}, p \chi_{x}\right)_{\Omega_{w}}\right| & \leq C \delta_{w} \varepsilon^{-1 / 2} N^{-1}(\ln N)\left\|\chi_{x}\right\|_{0, \Omega_{w}} \\
& \leq C \delta_{w} \varepsilon^{-1} N^{-1}(\ln N)\|\chi\|_{\varepsilon} .
\end{aligned}
$$

Combining these inequalities with (6.5), we have

$$
\left|a_{s t a b}\left(u-u^{I}, \chi\right)_{\Omega_{w}}\right| \leq C\left[\delta_{w} \varepsilon^{-1}+\delta_{w}^{1 / 2} N^{-2}(\ln N)\right]\|\chi\|_{S D} \quad \text { for all } \chi \in V^{N} .
$$


6.3. Subdomains $\Omega_{n}$ and $\Omega_{s}$. In this section we shall need the inequality

$$
\begin{aligned}
\delta_{s}^{1 / 4} \varepsilon^{1 / 4}\left\|\chi_{x}\right\|_{0, \Omega_{s}} & =\left(\delta_{s}^{1 / 4}\left\|\chi_{x}\right\|_{0, \Omega_{s}}^{1 / 2}\right)\left(\varepsilon^{1 / 4}\left\|\chi_{x}\right\|_{0, \Omega_{s}}^{1 / 2}\right) \\
& \leq\left(\delta_{s}^{1 / 2}\left\|\chi_{x}\right\|_{0, \Omega_{s}}+\varepsilon^{1 / 2}\left\|\chi_{x}\right\|_{0, \Omega_{s}}\right) / 2 \leq\|\| \chi \|_{S D} .
\end{aligned}
$$

Now $\left(\Delta v, p \chi_{x}\right)_{\Omega_{s}}+\left(\Delta v, p \chi_{x}\right)_{\Omega_{s w}}=-\left((p \Delta v)_{x}, \chi\right)_{\Omega_{s} \cup \Omega_{s w}}$ so by (2.2a) and the Cauchy-Schwarz inequality one has

$$
\begin{aligned}
\delta_{s} \varepsilon\left|\left(\Delta v, p \chi_{x}\right)_{\Omega_{s}}\right| & \leq C \delta_{s} \varepsilon\left(\left|\Omega_{s} \cup \Omega_{s w}\right|^{1 / 2}\|\chi\|_{0, \Omega_{s} \cup \Omega_{s w}}+\left|\Omega_{s w}\right|^{1 / 2}\left\|\chi_{x}\right\|_{0, \Omega_{s w}}\right) \\
& \leq C \delta_{s} \varepsilon\|\chi\| \|_{\varepsilon},
\end{aligned}
$$

where we used (3.6). For the convection term, Lemma 6.1, (2.2a) and (6.8) yield

$$
\begin{aligned}
\delta_{s}\left|\left(p\left(v-v^{I}\right)_{x}, p \chi_{x}\right)_{\Omega_{s}}\right| \leq & C \delta_{s} N^{-2}\left[\left\|v_{x x}\right\|_{0, \Omega_{s}}+\varepsilon^{1 / 2}(\ln N)\left\|v_{x y}\right\|_{0, \Omega_{s}}\right. \\
& \left.+\varepsilon(\ln N)^{2}\left\|v_{x y y}\right\|_{0, \Omega_{s}}\right]\left\|\chi_{x}\right\|_{0, \Omega_{s}} \\
\leq & \leq \delta_{s} \varepsilon^{1 / 4} N^{-2}(\ln N)\left\|\chi_{x}\right\|_{0, \Omega_{s}} \\
\leq & \leq \delta_{s}^{3 / 4} N^{-2}(\ln N)\|\chi\|_{S D} .
\end{aligned}
$$

Recall that $\eta_{1}=E+z_{00}+z_{01}$. By (2.2), Lemma 6.2 and (6.8) one has

$$
\begin{aligned}
\delta_{s} \varepsilon\left|\left(\Delta \eta_{1}, p \chi_{x}\right)_{\Omega_{s}}\right| & \leq \delta_{s} \varepsilon\left\|\Delta \eta_{1}\right\|_{L_{1}\left(\Omega_{s}\right)}\left\|\chi_{x}\right\|_{L_{\infty}\left(\Omega_{s}\right)} \\
& \leq C \delta_{s} \varepsilon N^{-5 / 2} \varepsilon^{-1 / 2}(\ln N)\left[\varepsilon^{1 / 2} N^{-2}(\ln N)\right]^{-1 / 2}\left\|\chi_{x}\right\|_{0, \Omega_{s}} \\
& \leq C \delta_{s}^{3 / 4} N^{-3 / 2}(\ln N)^{1 / 2}\|\chi\|_{S D}
\end{aligned}
$$

and

$$
\begin{aligned}
\delta_{s}\left|\left(p\left(\eta_{1}-\eta_{1}^{I}\right)_{x}, p \chi_{x}\right)_{\Omega_{s}}\right| & \leq C \delta_{s}\left(\left\|\eta_{1, x}\right\|_{L_{1}\left(\Omega_{s}\right)}\left\|\chi_{x}\right\|_{L_{\infty}\left(\Omega_{s}\right)}+\left\|\eta_{1, x}^{I}\right\|_{0, \Omega_{s}}\left\|\chi_{x}\right\|_{0, \Omega_{s}}\right) \\
& \leq C \delta_{s} N\left[\left\|\eta_{1, x}\right\|_{L_{1}\left(\Omega_{s}\right)} \varepsilon^{-1 / 4}(\ln N)^{-1 / 2}\left\|\chi_{x}\right\|_{0, \Omega_{s}}\right. \\
& \left.+\varepsilon^{1 / 4}(\ln N)^{1 / 2}\left\|\eta_{1}^{I}\right\|_{L_{\infty}\left(\Omega_{s}\right)}\left\|\chi_{x}\right\|_{0, \Omega_{s}}\right] \\
& \leq C \delta_{s}^{3 / 4} N^{-3 / 2}(\ln N)^{1 / 2}\|\| \|_{S D} .
\end{aligned}
$$

Recall that $\eta_{2}=z_{10}+z_{11}$ and that $D_{10}^{\varepsilon} \subset \Omega$ is the quarter disc with centre $(1,0)$ and radius $\varepsilon$. Now

$$
\left\|\Delta \eta_{2}\right\|_{0, \Omega_{s}} \leq\left\|\Delta \eta_{2}\right\|_{0, D_{10}^{\varepsilon}}+\left\|\Delta \eta_{2}\right\|_{0, \Omega_{s} \backslash D_{10}^{\varepsilon}} \leq C\left(|\ln \varepsilon|+\varepsilon^{-3 / 4}\right) \leq C \varepsilon^{-3 / 4}
$$

using (2.2d), (2.2f) and (2.2g). Hence, invoking (6.8),

$$
\delta_{s} \varepsilon\left|\left(\Delta \eta_{2}, p \chi_{x}\right)_{\Omega_{s}}\right| \leq C \delta_{s} \varepsilon\left\|\Delta \eta_{2}\right\|_{0, \Omega_{s}}\left\|\chi_{x}\right\|_{0, \Omega_{s}} \leq C \delta_{s} \varepsilon^{1 / 4}\left\|\chi_{x}\right\|_{0, \Omega_{s}} \leq C \delta_{s}^{3 / 4}\|\|_{\|_{S D}} .
$$

Furthermore,

$$
\begin{aligned}
\delta_{s}\left|\left(p\left(\eta_{2}-\eta_{2}^{I}\right)_{x}, p \chi_{x}\right)_{\Omega_{s}}\right| & \leq C \delta_{s}\left\|\left(\eta_{2}-\eta_{2}^{I}\right)_{x}\right\|_{0, \Omega_{s}}\left\|\chi_{x}\right\|_{0, \Omega_{s}} \\
& \leq C \delta_{s}\left|\Omega_{s}\right|^{1 / 2}\left\|\left(\eta_{2}-\eta_{2}^{I}\right)_{x}\right\|_{L_{\infty}\left(\Omega_{s}\right)}\left\|\chi_{x}\right\|_{0, \Omega_{s}} \\
& \leq C \delta_{s} \varepsilon^{1 / 4}(\ln N)^{1 / 2}\left\|\eta_{2, x}\right\|_{L_{\infty}\left(\Omega_{s}\right)}\left\|\chi_{x}\right\|_{0, \Omega_{s}} \\
& \leq C \delta_{s}^{3 / 4}(\ln N)^{1 / 2}\|\chi\|_{S D},
\end{aligned}
$$

where we used (2.2d), (2.2f), (2.2g) and (6.8).

By symmetry, similar estimates are valid on $\Omega_{n}$, where we assume that $\delta_{n}=\delta_{s}$. 
Combining these inequalities with (6.5), we have

$$
\left|a_{s t a b}\left(u-u^{I}, \chi\right)_{\Omega_{s} \cup \Omega_{n}}\right| \leq C\left[\delta_{s} \varepsilon+\delta_{s}^{3 / 4}(\ln N)^{1 / 2}+\delta_{s}^{1 / 2} N^{-2}(\ln N)\right]\|\| \chi \|_{S D}
$$

for all $\chi \in V^{N}$.

6.4. Subdomains $\Omega_{n w}$ and $\Omega_{s w}$. Recall that $\eta_{3}=v+E$. The Cauchy-Schwarz inequality, (2.2a) and (2.2b) yield

$$
\delta_{s w} \varepsilon\left|\left(\Delta \eta_{3}, p \chi_{x}\right)_{\Omega_{s w}}\right| \leq C \delta_{s w} \varepsilon^{-1 / 4}\left\|\chi_{x}\right\|_{0} \leq C \delta_{s w} \varepsilon^{-3 / 4}\|\| \chi \|_{\varepsilon},
$$

and by Lemma 6.1 and a variant of (6.8),

$$
\begin{aligned}
\delta_{s w}\left|\left(p\left(\eta_{3}-\eta_{3}^{I}\right)_{x}, \chi_{x}\right)_{\Omega_{s w}}\right| & \leq C \delta_{s w} \varepsilon^{1 / 4} N^{-2}(\ln N)^{2}\left\|\chi_{x}\right\|_{0, \Omega_{s w}} \\
& \leq C \delta_{s w}^{3 / 4} N^{-2}(\ln N)^{2}\|\chi\|_{S D} .
\end{aligned}
$$

Recall that $\eta_{4}=z_{00}+z_{01}+z_{10}+z_{11}$ and $D_{00}^{\varepsilon} \subset \Omega$ is the quarter disc with centre $(0,0)$ and radius $\varepsilon$. Now

$$
\left\|\Delta \eta_{4}\right\|_{0, \Omega_{s w}} \leq\left\|\Delta \eta_{2}\right\|_{0, D_{00}^{\varepsilon}}+\left\|\Delta \eta_{2}\right\|_{0, \Omega_{s w} \backslash D_{00}^{\varepsilon}} \leq C\left(|\ln \varepsilon|+\varepsilon^{-5 / 4}\right) \leq C \varepsilon^{-5 / 4}
$$

using (2.2). Hence

$$
\delta_{s w} \varepsilon\left|\left(\Delta \eta_{4}, p \chi_{x}\right)_{\Omega_{s w}}\right| \leq C \delta_{s w} \varepsilon\left\|\Delta \eta_{4}\right\|_{0, \Omega_{s w}}\left\|\chi_{x}\right\|_{0, \Omega_{s w}} \leq C \delta_{s w} \varepsilon^{-3 / 4}\|\| \|_{S D}
$$

Also, using (2.2) and Lemma 6.2 we get

$$
\begin{aligned}
\delta_{s w}\left|\left(p\left(\eta_{4}-\eta_{4}^{I}\right)_{x}, p \chi_{x}\right)_{\Omega_{s w}}\right| & \leq C \delta_{s w}\left\|\left(\eta_{4}-\eta_{4}^{I}\right)_{x}\right\|_{0, \Omega_{s w}}\left\|\chi_{x}\right\|_{0, \Omega_{s w}} \\
& \leq C \delta_{s w}\left|\Omega_{s w}\right|^{1 / 2}\left\|\eta_{4, x}\right\|_{L_{\infty}\left(\Omega_{s w}\right)}\left\|\chi_{x}\right\|_{0, \Omega_{s w}} \\
& \leq C \delta_{s w} \varepsilon^{-1 / 4}(\ln N)\|\chi\|_{S D} .
\end{aligned}
$$
$\delta_{s w}$.

By symmetry similar estimates are valid on $\Omega_{n w}$, where we assume that $\delta_{n w}=$

Combining these inequalities with 6.5 , we have

$$
\begin{aligned}
\mid a_{s t a b}(u- & \left.u^{I}, \chi\right)_{\Omega_{s w} \cup \Omega_{n w}} \mid \\
& \leq C\left[\delta_{s w} \varepsilon^{-3 / 4}+\delta_{s w}^{3 / 4} N^{-2}(\ln N)^{2}+\delta_{s w}^{1 / 2} N^{-2}\right]\|\chi \mid\|_{S D}
\end{aligned}
$$

for all $\chi \in V^{N}$.

6.5. Error bound for SDFEM solution. We will now use the bounds derived so far for $\left|a_{\text {stab }}\left(u-u^{I}, \chi\right)_{\Omega_{*}}\right|$ in each mesh region $\Omega_{*}$ to make good choices for the SDFEM parameters $\delta_{*}$. Increasing $\delta_{*}$ strengthens the norm $\|\cdot \cdot\| \|_{S D}$ in which we derive an error bound, but increasing it too much will decrease the order of convergence attained. Our main result (Theorem 6.3) restricts the $\delta_{*}$ to ensure almost first-order convergence, which is the highest order that we can prove in our analysis.

Theorem 6.3. Let $w^{N}$ be the SDFEM solution defined in (6.1). Let $\theta \in[1,2]$ be arbitrary. If we choose

$$
\begin{aligned}
& \delta_{e} \leq C \min \left\{N^{1-\theta}, \varepsilon^{-1 / 2} N^{-\theta}\right\}, \quad \delta_{w} \leq C \varepsilon N^{-\theta}, \\
& \delta_{n}=\delta_{s} \leq C N^{-4 \theta / 3}, \quad \delta_{n w}=\delta_{s w} \leq C \varepsilon^{3 / 4} N^{-\theta},
\end{aligned}
$$

then

$$
\left\|w^{N}-u^{I}\right\|_{S D} \leq C N^{-1} \ln N
$$


and

$$
\left\|w^{N}-u\right\|_{\varepsilon} \leq C N^{-1} \ln N
$$

Proof. Adding the bounds (6.6), 6.7), (6.9) and (6.10), and observing in the case of (6.9) that $\min \left\{\varepsilon^{-1} N^{-\theta}, N^{-4 \theta / 3}\right\}=N^{-4 \theta / 3}$, we get

$$
\left|a_{s t a b}\left(u-u^{I}, \chi\right)\right| \leq C\left(N^{-1} \ln N\right)^{\theta} \mid\|\chi\|_{S D} \quad \text { for all } \chi \in V^{N} .
$$

This inequality, (6.3) and (6.4) give the desired bound on $\left\|w^{N}-u^{I} \mid\right\|_{S D}$. The bound on $\left\|\mid w^{N}-u\right\| \|_{\varepsilon}$ then follows from Theorem 4.3 and a triangle inequality.

Remark 6.4. The values prescribed for the SD parameters in [4], where no corner singularities appear, are $\delta_{e} \leq C N^{-1}$ (when $\varepsilon \leq N^{-1}$, as we assumed in (3.6)), $\delta_{w} \leq C \varepsilon N^{-2}, \delta_{n} \leq C \varepsilon^{-1 / 4} N^{-2}$ and $\delta_{n w} \leq C \varepsilon^{3 / 4} N^{-2}$. These agree with our choices of $\delta_{e}$ (provided that $\varepsilon \leq N^{-2}$ ), $\delta_{w}$ and $\delta_{n w}$ when $\theta=2$. Our different choice of $\delta_{n}$ is caused by the inflow corner singularities.

\section{NumericAl RESUltS}

It is difficult to construct an example that satisfies exactly the hypotheses of our paper yet whose solution $u$ is known explicitly. Thus to conduct numerical experiments into the accuracy of our Galerkin method we use a double-mesh principle 2] where the computed solution on a given mesh is compared with the computed solution on a finer mesh.

Let $S^{N}$ denote the Shishkin mesh of Section 3, which has $N$ mesh intervals in each coordinate direction with transition points specified by (3.1). Let $\tilde{S}^{2 N}$ denote a modified Shishkin mesh that is piecewise equidistant with $2 N$ mesh intervals in each coordinate direction but with the transition points still specified by (3.1); that is, we do not change $N$ to $2 N$ in (3.1) so $\tilde{S}^{2 N} \neq S^{2 N}$. The mesh $\tilde{S}^{2 N}$ can equivalently be obtained by bisecting each mesh interval in $S^{N}$.

We introduce $\tilde{S}^{2 N}$ since piecewise bilinear functions on $S^{N}$ and $\tilde{S}^{2 N}$ can be compared more easily than piecewise bilinears on $S^{N}$ and $S^{2 N}$. From the theoretical point of view $\tilde{S}^{2 N}$ has the same convergence properties as $S^{2 N}$. Write $\tilde{u}^{2 N}$ for the Galerkin bilinear solution computed on the mesh $\tilde{S}^{2 N}$. Let $\left(\tilde{u}^{2 N}\right)^{I}$ denote the piecewise bilinear nodal interpolant of $\tilde{u}^{2 N}$ on the mesh $S^{N}$.

Our first example has constant data.

\section{Example 7.1.}

$$
\begin{aligned}
-\varepsilon \Delta u-u_{x}+2 u & =1 & & \text { in } \Omega, \\
u & =0 & & \text { on } \partial \Omega,
\end{aligned}
$$

The second example has variable data.

\section{Example 7.2.}

$$
\begin{gathered}
-\varepsilon \Delta u-(2-x)(1+y(1-y)) u_{x}+(3 / 2+\sin (\pi y)) u \\
=(2-x)(3 / 2-\sin (\pi y)) \text { in } \Omega, \\
u=0 \quad \text { on } \partial \Omega
\end{gathered}
$$


TABLE 1. Numerical errors for Galerkin method (5.1) applied to Example 7.1

\begin{tabular}{r||ll|ll}
$N$ & $\|\left|\tilde{u}^{2 N}-u^{N}\right|||$ & $\left\|\left(\tilde{u}^{2 N}\right)^{I}-u^{N}\right\| \|_{\varepsilon}$ \\
\hline 8 & $1.008 \mathrm{e}-1$ & 0.94 & $2.370 \mathrm{e}-2$ & 1.80 \\
16 & $6.886 \mathrm{e}-2$ & 0.97 & $1.144 \mathrm{e}-2$ & 1.89 \\
32 & $4.370 \mathrm{e}-2$ & 0.99 & $4.716 \mathrm{e}-3$ & 1.94 \\
64 & $2.641 \mathrm{e}-2$ & 0.99 & $1.752 \mathrm{e}-3$ & 1.97 \\
128 & $1.545 \mathrm{e}-2$ & 1.00 & $6.064 \mathrm{e}-4$ & 1.98 \\
256 & $8.839 \mathrm{e}-3$ & 1.00 & $2.000 \mathrm{e}-4$ & 1.99 \\
512 & $4.974 \mathrm{e}-3$ & & $6.367 \mathrm{e}-5$ &
\end{tabular}

TABLE 2. Numerical errors for Galerkin method (5.1) applied to Example 7.2

\begin{tabular}{r||ll|ll}
$N$ & $\left\||| \tilde{u}^{2 N}-u^{N}|\||_{\varepsilon}\right.$ & $\left\|\left(\tilde{u}^{2 N}\right)^{I}-u^{N}\right\| \|_{\varepsilon}$ \\
\hline 8 & $3.435 \mathrm{e}-1$ & 0.81 & $1.407 \mathrm{e}-1$ & 1.52 \\
16 & $2.472 \mathrm{e}-1$ & 0.92 & $7.589 \mathrm{e}-2$ & 1.78 \\
32 & $1.604 \mathrm{e}-1$ & 0.97 & $3.293 \mathrm{e}-2$ & 1.91 \\
64 & $9.778 \mathrm{e}-2$ & 0.99 & $1.242 \mathrm{e}-2$ & 1.96 \\
128 & $5.737 \mathrm{e}-2$ & 1.00 & $4.308 \mathrm{e}-3$ & 1.98 \\
256 & $3.285 \mathrm{e}-2$ & 1.00 & $1.421 \mathrm{e}-3$ & 1.98 \\
512 & $1.849 \mathrm{e}-2$ & & $4.536 \mathrm{e}-4$ &
\end{tabular}

The homogeneity of the Dirichlet boundary data and the property that $f \neq 0$ at each corner together ensure that the solution of Example 7.1 satisfies the estimates (2.1) and (2.2); see [5, 6]. For Example 7.2] no analogous pointwise a priori bounds on derivatives are known so, as in our theoretical analysis, we assume that the same estimates are satisfied.

Our numerical results will illustrate the convergence results of Theorem 5.3. Corollary 5.4 and Theorem 6.3 and also show the effect of choosing too large a value of $\theta$ in Theorem 6.3 .

Tables 1 and 2 give the numerical results when the Galerkin method (5.1) is used to solve these two examples. In each table the errors $\left|\left\|\tilde{u}^{2 N}-u^{N} \mid\right\| \|_{\varepsilon}\right.$ and $\left\|\left(\tilde{u}^{2 N}\right)^{I}-u^{N} \mid\right\|_{\varepsilon}$ shown are, for each value of $N$, the maximum error obtained when $\varepsilon$ takes the values $10^{-4}, 10^{-6}, 10^{-8}, 10^{-10}$. This is done to illustrate that the method is robust with respect to small $\varepsilon$. The convergence rate in each column entry is the value of $\alpha$ in $\mathcal{O}\left(\left(N^{-1} \ln N\right)^{\alpha}\right)$, where two successive vertical entries are used to compute each $\alpha$ in the usual way [2].

The errors for Example 7.2 are a little larger than those for Example 7.1 as one might expect, but it is clear that the method works equally well for both problems. From the convergence rates for $\left\|\left|\tilde{u}^{2 N}-u^{N}\right|\right\|_{\varepsilon}$ one sees that the $\mathcal{O}\left(N^{-1} \ln N\right)$ prediction of Corollary 5.4 is fulfilled. The $\left.\left\|\left(\tilde{u}^{2 N}\right)^{I}-u^{N}\right\|\right|_{\varepsilon}$ column, where secondorder convergence is apparent, exhibits a second-order supercloseness property at the discrete level that is superior to the first-order convergence of Theorem 5.3. Whether this supercloseness is due to the double-mesh principle or whether Theorem 5.3 is not sharp is a topic for future research. 
TABLE 3. Numerical errors for SDFEM (6.1) applied to Example 7.2 with $\theta=1.5$

\begin{tabular}{r|llllllll}
$N$ & $\left\|\left|\tilde{w}^{2 N}-w^{N}\right|\right\|_{\varepsilon}$ & & $\left\|\tilde{w}^{2 N}-w^{N} \mid\right\|_{S D}$ & & & $\left\|\left(\tilde{w}^{2 N}\right)^{I}-w^{N}\right\| \|_{\varepsilon}$ & & $\left\|\left(\tilde{w}^{2 N}\right)^{I}-w^{N}\right\| \|_{S D}$ \\
\hline 8 & $2.993 \mathrm{e}-1$ & 0.62 & $3.381 \mathrm{e}-1$ & 0.80 & $8.183 \mathrm{e}-2$ & 0.89 & $1.101 \mathrm{e}-1$ & 1.20 \\
16 & $2.330 \mathrm{e}-1$ & 0.85 & $2.447 \mathrm{e}-1$ & 0.91 & $5.698 \mathrm{e}-2$ & 1.36 & $6.765 \mathrm{e}-2$ & 1.49 \\
32 & $1.564 \mathrm{e}-1$ & 0.94 & $1.599 \mathrm{e}-1$ & 0.97 & $3.003 \mathrm{e}-2$ & 1.60 & $3.354 \mathrm{e}-2$ & 1.70 \\
64 & $9.669 \mathrm{e}-2$ & 0.98 & $9.755 \mathrm{e}-2$ & 0.99 & $1.328 \mathrm{e}-2$ & 1.71 & $1.410 \mathrm{e}-2$ & 1.71 \\
128 & $5.701 \mathrm{e}-2$ & 0.99 & $5.732 \mathrm{e}-2$ & 1.00 & $5.273 \mathrm{e}-3$ & 1.67 & $5.598 \mathrm{e}-3$ & 1.72 \\
256 & $3.276 \mathrm{e}-2$ & 1.00 & $3.284 \mathrm{e}-2$ & 1.00 & $2.069 \mathrm{e}-3$ & 1.69 & $2.142 \mathrm{e}-3$ & 1.73 \\
512 & $1.847 \mathrm{e}-2$ & & $1.849 \mathrm{e}-2$ & & $7.824 \mathrm{e}-4$ & & $7.938 \mathrm{e}-4$ &
\end{tabular}

TABLE 4. Numerical errors for SDFEM (6.1) applied to Example 7.2 with $\theta=2.0$

\begin{tabular}{r|llllllll}
$N$ & $\left\|\left|\tilde{w}^{2 N}-w^{N}\right|\right\|_{\varepsilon}$ & & $\left\|\tilde{w}^{2 N}-w^{N}\right\|_{S D}$ & & $\left\|\left(\tilde{w}^{2 N}\right)^{I}-w^{N}\right\| \|_{\varepsilon}$ & $\left\|\left(\tilde{w}^{2 N}\right)^{I}-w^{N}\right\|_{S D}$ \\
\hline 8 & $3.280 \mathrm{e}-1$ & 0.74 & $3.439 \mathrm{e}-1$ & 0.82 & $1.137 \mathrm{e}-1$ & 1.22 & $1.226 \mathrm{e}-1$ & 1.35 \\
16 & $2.434 \mathrm{e}-1$ & 0.90 & $2.463 \mathrm{e}-1$ & 0.92 & $6.928 \mathrm{e}-2$ & 1.68 & $7.091 \mathrm{e}-2$ & 1.71 \\
32 & $1.593 \mathrm{e}-1$ & 0.96 & $1.601 \mathrm{e}-1$ & 0.97 & $3.146 \mathrm{e}-2$ & 1.87 & $3.169 \mathrm{e}-2$ & 1.88 \\
64 & $9.749 \mathrm{e}-2$ & 0.99 & $9.770 \mathrm{e}-2$ & 0.99 & $1.210 \mathrm{e}-2$ & 1.95 & $1.213 \mathrm{e}-2$ & 1.95 \\
128 & $5.732 \mathrm{e}-2$ & 1.00 & $5.736 \mathrm{e}-2$ & 1.00 & $4.236 \mathrm{e}-3$ & 1.97 & $4.239 \mathrm{e}-3$ & 1.97 \\
256 & $3.284 \mathrm{e}-2$ & 1.00 & $3.285 \mathrm{e}-2$ & 1.00 & $1.403 \mathrm{e}-3$ & 1.98 & $1.404 \mathrm{e}-3$ & 1.98 \\
512 & $1.849 \mathrm{e}-2$ & & $1.849 \mathrm{e}-2$ & & $4.491 \mathrm{e}-4$ & & $4.491 \mathrm{e}-4$ &
\end{tabular}

TABLE 5. Numerical errors for SDFEM (6.1) applied to Example 7.2 with $\theta=2.5$

\begin{tabular}{r|lllllllc}
$N$ & \|\|$\tilde{w}^{2 N}-w^{N} \mid\|\|_{\varepsilon}$ & & $\left\|\tilde{w}^{2 N}-w^{N}\right\| \|_{S D}$ & & $\left\|\left(\tilde{w}^{2 N}\right)^{I}-w^{N}\right\| \|_{\varepsilon}$ & $\left\|\left(\tilde{w}^{2 N}\right)^{I}-w^{N}\right\|_{S D}$ \\
\hline 8 & $3.382 \mathrm{e}-1$ & 0.79 & $3.440 \mathrm{e}-1$ & 0.82 & $1.284 \mathrm{e}-1$ & 1.38 & $1.315 \mathrm{e}-1$ & 1.42 \\
16 & $2.459 \mathrm{e}-1$ & 0.91 & $2.469 \mathrm{e}-1$ & 0.92 & $7.351 \mathrm{e}-2$ & 1.74 & $7.382 \mathrm{e}-2$ & 1.75 \\
32 & $1.602 \mathrm{e}-1$ & 0.97 & $1.604 \mathrm{e}-1$ & 0.97 & $3.246 \mathrm{e}-2$ & 1.90 & $3.249 \mathrm{e}-2$ & 1.90 \\
64 & $9.774 \mathrm{e}-2$ & 0.99 & $9.777 \mathrm{e}-2$ & 0.99 & $1.233 \mathrm{e}-2$ & 1.96 & $1.233 \mathrm{e}-2$ & 1.96 \\
128 & $5.737 \mathrm{e}-2$ & 1.00 & $5.737 \mathrm{e}-2$ & 1.00 & $4.292 \mathrm{e}-3$ & 1.98 & $4.293 \mathrm{e}-3$ & 1.98 \\
256 & $3.285 \mathrm{e}-2$ & 1.00 & $3.285 \mathrm{e}-2$ & 1.00 & $1.419 \mathrm{e}-3$ & 1.98 & $1.419 \mathrm{e}-3$ & 1.98 \\
512 & $1.849 \mathrm{e}-2$ & & $1.849 \mathrm{e}-2$ & & $4.532 \mathrm{e}-4$ & & $4.532 \mathrm{e}-4$ &
\end{tabular}

Next we move on to the SDFEM defined in (6.1). Let $\tilde{w}^{2 N}$ be the SDFEM solution on the mesh $\tilde{S}^{2 N}$. Tables 3 and 4 give results when this method is applied to Example 7.2 with $\theta=1.5$ and $\theta=2.0$, respectively. Here the $\delta_{*}$ are such that the upper bounds assumed in Theorem 6.3 are satisfied with $C=1$ in each case. We see from the tables that this change in the value of $\theta$ affects $u^{I}-w^{N}$, but not $u-w^{N}$. Increasing $\theta$ still further, to 2.5 , has little apparent effect on the numerical results - compare Tables 4 and 5 - but now the SD norm is weaker. 


\section{Conclusions}

The problem at hand contains boundary layers of two types: exponential and characteristic; and corner singularities of two types: incoming and outgoing. We constructed a piecewise-uniform Shishkin mesh that is refined so that boundary layers are resolved, but the mesh is not modified to address the corner singularities. On this mesh, Galerkin and streamline diffusion finite element methods with piecewise bilinears were examined. We gave error analyses for these methods that took account of both the boundary layers and the corner singularities. This analysis yielded an error bound of order $N^{-1} \ln N$ in various norms, where $N$ is the number of mesh intervals in each coordinate direction. As no example is available with a known exact solution that exhibits the precise solution behaviour predicted by theoretical bounds on derivatives, we carried out numerical tests using a "doublemesh principle" to study the solution error and rate of convergence. The tests show clearly the expected $\mathcal{O}\left(N^{-1} \ln N\right)$ convergence rate.

\section{ACKNOWLEDGMENT}

We thank the referees for their constructive criticisms of this paper.

\section{REFERENCES}

[1] T. Apel, Anisotropic finite elements: local estimates and applications, Advances in Numerical Mathematics, B. G. Teubner, Stuttgart, 1999. MR1716824(2000k:65002)

[2] P. A. Farrell, A. F. Hegarty, J. J. H. Miller, E. O'Riordan, and G. I. Shishkin, Robust computational techniques for boundary layers, Applied Mathematics (Boca Raton), vol. 16, Chapman \& Hall/CRC, Boca Raton, FL, 2000. MR.1750671 (2001c:65003)

[3] S. Franz and T. Linß, Superconvergence analysis of the Galerkin FEM for a singularly perturbed convection-diffusion problem with characteristic layers, Numer. Methods Partial Differential Equations 24 (2008), no. 1, 144-164. MR.2371352 (2008k:35015)

[4] S. Franz, T. Linß, and H.-G. Roos, Superconvergence analysis of the SDFEM for elliptic problems with characteristic layers, Appl. Numer. Math. 58 (2008), no. 12, 1818-1829. MR.2464813 (2009j:65316)

[5] R. B. Kellogg and M. Stynes, Corner singularities and boundary layers in a simple convection-diffusion problem, J. Differential Equations 213 (2005), no. 1, 81-120. MR2139339 (2006b:35069)

[6] _ Sharpened bounds for corner singularities and boundary layers in a simple convection-diffusion problem, Appl. Math. Lett. 20 (2007), no. 5, 539-544. MR2303990 (2007k:35093)

[7] Q. Lin, A rectangle test for finite element analysis, Proc. Syst. Sci. Eng., Great Wall (H.K.) Culture Publish Co., 1991, pp. 213-216.

[8] T. Linß, Uniform superconvergence of a Galerkin finite element method on Shishkintype meshes, Numer. Methods Partial Differential Equations 16 (2000), no. 5, 426-440. MR1778398 (2001e:65183)

[9] T. Linß and M. Stynes, Numerical methods on Shishkin meshes for linear convection-diffusion problems, Comput. Methods Appl. Mech. Engrg. 190 (2001), 3527-3542.

[10] H.-G. Roos, M. Stynes, and L. Tobiska, Robust numerical methods for singularly perturbed differential equations, second ed., Springer Series in Computational Mathematics, vol. 24, Springer-Verlag, Berlin, 2008, Convection-diffusion-reaction and flow problems. MR 2454024 (2009f:65002)

[11] G. I. Shishkin and L. P. Shishkina, Difference methods for singular perturbation problems, Chapman \& Hall/CRC Monographs and Surveys in Pure and Applied Mathematics, vol. 140, CRC Press, Boca Raton, FL, 2009. MR2454526 (2009j:65003) 
[12] M. Stynes and L. Tobiska, The SDFEM for a convection-diffusion problem with a boundary layer: optimal error analysis and enhancement of accuracy, SIAM J. Numer. Anal. 41 (2003), no. 5, 1620-1642. MR2035000(2004j:65193)

[13] Z. Zhang, Finite element superconvergence on Shishkin mesh for 2-d convection-diffusion problems, Math. Comp. 72 (2003), 1147-1177. MR.1972731(2004d:65130)

Department of Mathematics and Statistics, University of Limerick, Limerick, Ireland

E-mail address: sebastian.franz@ul.ie

Department of Mathematics, University of South Carolina, Columbia, South CarOLINA 29208

E-mail address: bklandrum@gmail.com

Department of Mathematics, National University of Ireland, Cork, Ireland

E-mail address: m.stynes@ucc.ie 